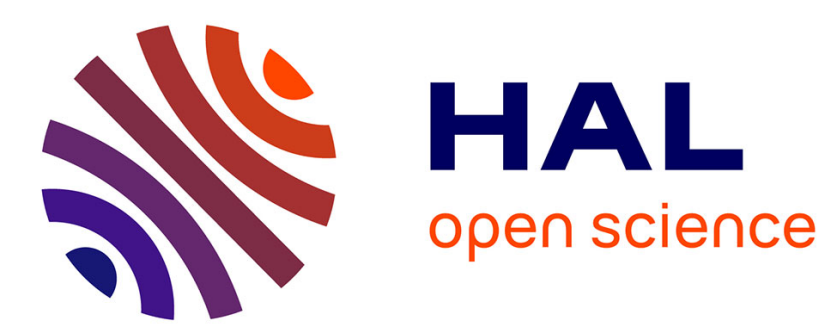

\title{
An extension of Pontryagin's principle for state-constrained optimal control of semilinear elliptic equations and variational inequalities
}

J. Frederic Bonnans, Eduardo Casas

\section{To cite this version:}

J. Frederic Bonnans, Eduardo Casas. An extension of Pontryagin's principle for state-constrained optimal control of semilinear elliptic equations and variational inequalities. [Research Report] RR1747, INRIA. 1992. inria-00076987

\section{HAL Id: inria-00076987 \\ https://hal.inria.fr/inria-00076987}

Submitted on 29 May 2006

HAL is a multi-disciplinary open access archive for the deposit and dissemination of scientific research documents, whether they are published or not. The documents may come from teaching and research institutions in France or abroad, or from public or private research centers.
L'archive ouverte pluridisciplinaire HAL, est destinée au dépôt et à la diffusion de documents scientifiques de niveau recherche, publiés ou non, émanant des établissements d'enseignement et de recherche français ou étrangers, des laboratoires publics ou privés. 


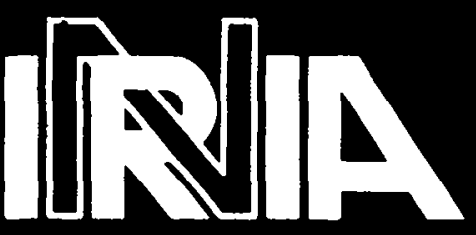

UNITÉ DE RECHERCHE INRIA-ROCQUENCOURT

\section{Rapports de Recherche}

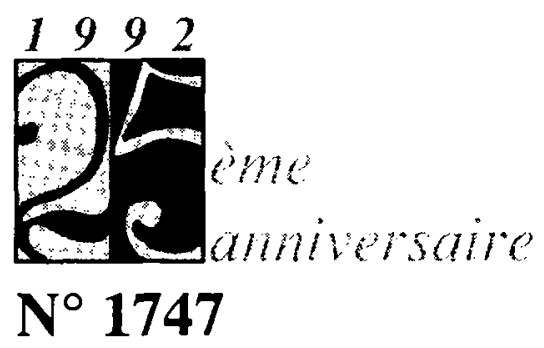

\section{Programme 5}

Traitement du Signal, Automatique et Productique
Institut National de Recherche en Informatique et en Automatique

Domaine de Voluceau Rocquencourt B.P.105

78153 Le Chesnay Cedex France Tél.:(1) 39635511
Frédéric BONNANS Eduardo CASAS 
Une extension du principe de Pontryagine pour le contrôle optimal avec contraintes sur l'état d'équations semilinéaires elliptiques et d'inéquations variationnelles

\author{
An extension of Pontryagin's principle for state-constrained \\ optimal control of semilinear elliptic equations and \\ variational inequalities ${ }^{1}$ \\ Frédéric BONNANS \\ INRIA, Domaine de Voluceau, BP 105, Rocquencourt \\ 78153 Le Chesnay, France \\ and \\ Eduardo CASAS \\ Departamento de Matemática Aplicada y Ciencias de la Computación \\ E.T.S.I. de Caminos, C. y P., Universidad de Cantabria \\ Av. Los Castros s/n., 39071 Santander, Spain
}

\title{
Résumé
}

Cet article traite des problèmes de contrôle optimal avec contraintes sur l'état gouvernés par des équations semilinéaires elliptiques ou des inéquations variationnelles. Utilisant le principe d'Ekeland, nous obtenons un principe du minimum de Pontryagine sous certaines conditions de stabilité du coût optimal par rapport aux contraintes sur l'état.

\begin{abstract}
This paper deals with state-constrained optimal control problems governed by semilinear elliptic equations or variational inequalities. By using Ekeland's principle, we derive a minimum principle of Pontryagin's type under some stability conditions of the optimal cost with respect to the state constraints.
\end{abstract}

\footnotetext{
${ }^{1}$ The research of the second author was partially supported by Direccion General de Investigación Científica y 'Técnica (Madrid)
} 


\title{
AN EXTENSION OF PONTRYAGIN'S PRINCIPLE FOR STATE-CONSTRAINED OPTIMAL CONTROL OF SEMILINEAR ELLIPTIC EQUATIONS AND VARIATIONAL INEQUALITIES*
}

\author{
FRÉDÉRIC BONNANS ${ }^{\dagger}$ AND EDUARDO CASAS ${ }^{\ddagger}$
}

\begin{abstract}
This paper deals with state-constrained optimal control problems governed by semilinear elliptic equations or variational inequalities. By using Ekeland's principle, we derive a minimum principle of Pontryagin's type under some stability conditions of the optimal cost with respect to the state constraints.
\end{abstract}

Key words. Pontryagin's principle, boundary control, semilinear elliptic operators, optimality conditions, state constraints

AMS(MOS) subject classifications. 49K20, 35J65, 35J 85

1. Introduction. There exists a vast literature devoted to Pontryagin's principle for optimal control problems governed by ordinary differential equations or evolution partial differential equations, but very few papers have considered the case of elliptic equations. A simple case corresponding to a linear equation was studied by Lions [18]. More recently, the authors have derived Pontryagin's principle for semilinear monotone elliptic equations in [7]. Here we extend the results of the last work by letting the existence of pointwise state constraints generalizing some preliminary results of Bonnans [3], [4]; see Bonnans and Casas [5] for a different approach to the optimality conditions of state-constrained control problems.

The difficulty of deriving the optimality conditions for control problems associated to variational inequalities is well known; see the works of Mignot [19], Mignot and Puel [20], Barbu [2]. Zheng-Xu He [17] obtained the optimality conditions for stateconstrained problems governed by variational inequalities and Bonnans and Tiba [8] proved Pontryagin's principle for control problems of semilinear elliptic variational inequalities. Here we will derive a principle of Pontryagin's type for state-constrained control problems of semilinear elliptic variational inequalities.

In this article we prove Pontryagin's principle as follows: with the aid of Ekeland's principle, we introduce a family of control problems without state constraints for which some approximate solutions converge towards the optimal control of the initial problem; we derive the optimality conditions for the problems of this family by using some results on problems without state constraints that generalize those of Bonnans and Casas [7] and Bonnans and Tiba [8] and finally we pass to the limit. In order to apply Ekeland's principle we need assume some stability conditions of the optimal cost with respect to small perturbations of the feasible state set. We distinguish two different stability

\footnotetext{
* The research of the second author was partially supported by Dirección General de Investigación Cientifica y Técnica (Madrid)

$\uparrow$ INRIA, Domaine de Voluceau, BP 105, Rocquencourt, 78153 Le Chesnay, France

$\$$ Departamento de Matemática Aplicada y Ciencias de la Computación, E.T.S.I. de Caminos, C. y P., Universidad de Cantabria, 39071 Santander, Spain.
} 
conditions, called weak and strong respectively. Under a weak stability condition we derive the optimality conditions in a non qualified form, while the strong stability allows to prove a qualified Pontryagin's principle. The weak stability condition has been used by Casas [12] to prove the convergence of the numerical approximations of statc-constrained control problems.

The paper is organized as follows: in the next section we formulate the control problem associated to a monotone semilinear elliptic equation and in Section 3 the statements of the weak and strong Pontryagin's principles are presented; in Section 4 we give some technical results used in sections 5 and 6 to prove the theorems stated in the third section; finally Section 7 is devoted to the control of variational inequalities.

2. Setting of the problem. Let $\Omega$ be an open and bounded subset of $R^{n}, n \geq 1$, with a Lipschitz boundary $\Gamma$. Given a non empty bounded set $K \subset R^{m}, m \geq 1$, and $f: \Omega \times R \times K \longrightarrow R$ we consider the following boundary value problem

$$
\left\{\begin{aligned}
A y=f(x, y(x), u(x)) & \text { in } \Omega \\
y=0 & \text { on } \Gamma,
\end{aligned}\right.
$$

where

$$
A y=-\sum_{i, j=1}^{n} \partial_{x_{j}}\left(a_{i j}(x) \partial_{x_{i}} y(x)\right)
$$

$$
\left\{\begin{array}{l}
a_{i j} \in C^{0,1}(\bar{\Omega}) \text { and } \\
\exists \Lambda>0 \text { such that } \sum_{i, j=1}^{n} a_{i j}(x) \xi_{i} \xi_{j} \geq \Lambda|\xi|^{2} \quad \forall \xi \in R^{n}, \forall x \in \Omega .
\end{array}\right.
$$

We recall that $C^{0, \alpha}(\bar{\Omega})$, with $\alpha \in(0,1]$, is the space of all continue functions in $\bar{\Omega}$ that satisfy the Hölder condition:

$$
\sup _{x_{1}, x_{2} \in \bar{\Omega}} \frac{\left|u\left(x_{2}\right)-u\left(x_{1}\right)\right|}{\left|x_{2}-x_{1}\right|^{\alpha}}<+\infty .
$$

Given two measurable functions $L: \Omega \times R \times K \longrightarrow R$ and $g: \bar{\Omega} \times R \longrightarrow R$, for every $\delta>0$ we formulate the control problem

$$
\left(P_{\mathcal{S}}\right)\left\{\begin{array}{l}
\min J(y, u)=\int_{\Omega} L(x, y(x), u(x)) d x \\
(y, u) \text { satisfies }(2.1), u(x) \in K \text { a.e. } x \in \Omega \text { and } g(x, y(x)) \leq \delta \forall x \in \Omega .
\end{array}\right.
$$

We will make the following assumptions on the functions defining the problem $\left(P_{\delta}\right)$ : $g \in C(\bar{\Omega} \times R), g, L$ and $f$ are differentiable with respect to the second variable for every $(x, u) \in \Omega \times K$ and there exist functions $M_{1} \in L^{s}(\Omega), s>n / 2$ and $s \geq 2, M_{2} \in L^{1}(\Omega)$ 
and $\eta$ increasing monotone verifying for every $(x, y, u) \in \Omega \times R \times k$

$$
\left\{\begin{array}{l}
|f(x, 0, u)|+\left|\frac{\partial f}{\partial y}(x, y, u)\right| \leq M_{1}(x)+\eta(|y|), \\
|L(x, 0, u)|+\left|\frac{\partial L}{\partial y}(x, y, u)\right| \leq M_{2}(x)+\eta(|y|), \\
\frac{\partial g}{\partial y} \in C(\bar{\Omega} \times R), \quad \frac{\partial f}{\partial y}(x, y, u) \leq 0 .
\end{array}\right.
$$

We will say that a control $u: \Omega \longrightarrow R^{m}$ is feasible if $u(x) \in K$ a.e. $x \in \Omega$ and the mapping $(x, y) \longrightarrow(f(x, y, u(x)), L(x, y, u(x)))$ is measurable in $\Omega \times R$. The set of feasible controls is denoted by $\mathcal{K}$. In this set we define the distance, called Ekeland's distance,

$$
d(u, v)=m(\{x \in \Omega: u(x) \neq v(x)\}),
$$

where $m$ denotes the Lebesgue measure. Adapting the proof of Ekeland [15] to our case it is easy to check that $(\mathcal{K}, d)$ is a complete metric space (the only difference is that we have to check the feasibility of the limit of a Cauchy sequence, which is immediate from the definition and the fact that a limit of measurable functions is measurable).

Under the previous hypotheses and thanks to the boundedness of $K$, we can deduce the following theorem

THEOREM 2.1. There exist constants $C_{1}>0$ and $\alpha \in(0,1)$ such that for every $u \in \mathcal{K}$ the equation (2.1) has a unique solution $y_{u} \in H_{0}^{1}(\Omega) \cap C^{0, \alpha}(\bar{\Omega})$ satisfying

$$
\left\|y_{u}\right\|_{H_{0}^{1}(\Omega)}+\left\|y_{u}\right\|_{C^{0, \alpha}(\bar{\Omega})} \leq C_{1} .
$$

Furthermore the mapping $u \in(\mathcal{K}, d) \longrightarrow y_{u} \in H_{0}^{1}(\Omega) \cap C^{0, \alpha}(\bar{\Omega})$ is continuous.

Before proving this theorem we state the following lemma

LEMMA 2.2. There exist $\alpha \in(0,1), C_{2}$ and $C_{3}$ such that for every $a, b \in L^{s}(\Omega)$, $a(x) \geq 0$, the problem

$$
\begin{cases}A y+a y=b & \text { in } \Omega, \\ y=0 & \text { on } \Gamma,\end{cases}
$$

has a unique solution $y \in H_{0}^{1}(\Omega) \cap C^{0, \alpha}(\bar{\Omega})$ verifying

$$
\begin{gathered}
\|y\|_{H_{0}^{1}(\Omega)}+\|y\|_{L^{\infty}(\Omega)} \leq C_{2}\|b\|_{L^{\prime}(\Omega)}, \\
\|y\|_{C^{0, \alpha}(\bar{\Omega})} \leq C_{3}\|b\|_{L^{s}(\Omega)}\left(1+\|a\|_{L^{\prime}(\Omega)}\right)
\end{gathered}
$$

This result follows from classical estimations in the spaces $C^{0, \alpha}(\bar{\Omega})$ (Gilbarg and Trudinger [16] or Stampacchia [21]); see Bonnans and Casas [7] for details. Now we prove Theorem 2.1 . 
Proof. The first part of the theorem is also proved in Bonnans and Casas [7]. Let us prove the continuity of $u \longrightarrow y_{u}$. Let $\left\{u_{k}\right\}_{k=1}^{\infty} \subset \mathcal{K}$ be a sequence converging to $u \in \mathcal{K}$, i.e. $d\left(u_{k}, u\right) \rightarrow 0$. Denote by $y_{k}$ and $y$ the states corresponding to $u_{k}$ and $u$ respectively. From (2.3) and (2.4) we deduce the existence of $M>0$ such that

$$
b_{k}(x)=f\left(x, y_{k}(x), u(x)\right)-f\left(x, y_{k}(x), u_{k}(x)\right)
$$

satisfies

$$
\begin{gathered}
\left\|b_{k}\right\|_{L^{\prime}(\Omega)}= \\
\left(\int_{\left\{x: u_{k}(x) \neq u(x)\right\}}\left|f\left(x, y_{k}(x), u(x)\right)-f\left(x, y_{k}(x), u_{k}(x)\right)\right|^{s} d x\right)^{1 / s} \rightarrow 0 .
\end{gathered}
$$

Now applying the mean value theorem we get for some function $\theta_{k}: \bar{\Omega} \longrightarrow(0,1)$

$$
A\left(y-y_{k}\right)-\frac{\partial f}{\partial y}\left(x, y+\theta_{k}\left(y-y_{k}\right), u(x)\right)\left(y-y_{k}\right)=b_{k} .
$$

Therefore, from Lemma 2.2 and using (2.3) we obtain

$$
\left\|y-y_{k}\right\|_{H_{0}^{1}(\Omega)}+\left\|y-y_{k}\right\|_{C^{0, \alpha}(\bar{\Omega})} \leq M^{\prime}\left\|b_{k}\right\|_{L^{s}(\Omega)} \rightarrow 0,
$$

which completes the proof.

REMARK 2.3. In order to use Lemma 2.2 in the above proof we have to check that

$$
x \longrightarrow \frac{\partial f}{\partial y}\left(x, y(x)+\theta_{k}(x)\left(y(x)-y_{k}(x)\right), u(x)\right)
$$

is a measurable function. This is true because by definition of $\theta_{k}$

$$
\begin{gathered}
\frac{\partial f}{\partial y}\left(x, y(x)+\theta_{k}(x)\left(y(x)-y_{k}(x)\right), u(x)\right)= \\
\left\{\begin{array}{cc}
\frac{f(x, y(x), u(x))-f\left(x, y_{k}(x), u(x)\right)}{y(x)-y_{k}(x)} & \text { if } y(x) \neq y_{k}(x), \\
\frac{\partial f}{\partial y}(x, y(x), u(x)) & \text { if } y(x)=y_{k}(x) .
\end{array}\right.
\end{gathered}
$$

We finish this section by proving a lemma that will be used several times in this paper. First let us introduce some notation. In the sequel $M(\Omega)$ will denote the space of real regular Borel measures in $\Omega$, which is identified with the dual space of $C_{0}(\Omega)$, space formed by the real continuous functions defined in $\bar{\Omega}$ and vanishing on $\Gamma$. Let $A^{*}$ denote the formal adjoint operator of $A$ :

$$
A^{*} y=-\sum_{i, j=1}^{n} \partial_{2, j}\left(a_{j i}(x) \partial_{x,} y(x)\right)
$$


Lrmm 2.4. For every function $a \in L^{s}(\Omega)$, with $a(x) \geq 0$ a.e. $x \in \Omega$, and every Borcl measure $\mu \in M(\Omega)$ there exists a unique solution in $W_{0}^{1, \sigma}(\Omega)$, for all $\sigma<n /(n-1)$, of problem

$$
\begin{cases}A^{\star} p+a p=\mu & \text { in } \Omega \\ y=0 & \text { on } \Gamma .\end{cases}
$$

Moreover there exists a constant $M>0$ independent of a such that

$$
\|p\|_{W_{0}^{1, \sigma}(\Omega)} \leq M\|\mu\|_{M(\Omega)}
$$

Proof. The existence and uniqueness in $W_{0}^{1, \sigma}(\Omega)$ of $p$ solution of the above Dirichlet problem is well known; see Stampacchia [21] or Casas [11]. Let us prove (2.8). Let $t$ be the conjugate of $\sigma, 1 / t+1 / \sigma=1$, thus $t>n$. For every $\psi \in W^{-1, t}(\Omega)=\left(W_{0}^{1, s}(\Omega)\right)^{\prime}$, the equation

$$
\begin{cases}A \varphi+a \varphi=\psi & \text { in } \Omega, \\ \varphi=0 & \text { on } \Gamma\end{cases}
$$

has a unique solution in $H_{0}^{1}(\Omega) \cap C_{0}(\Omega)$ and, proceeding as in [7, Lemma 3.2], there exists $M>0$ independent of $a$ such that

$$
\|\varphi\|_{\infty} \leq M\|\psi\|_{W-1, t(\Omega)}
$$

Hence

$$
\begin{gathered}
\left|\int_{\Omega} p \psi d x\right|=\left|\int_{\Omega} p(A \varphi+a \varphi) d x\right|=\left|\int_{\Omega} \varphi d \mu\right| \leq \\
\|\varphi\|_{\infty}\|\mu\|_{M(\Omega)} \leq M\|\psi\|_{W-1, t(\Omega)}\|\mu\|_{M(\Omega)},
\end{gathered}
$$

which proves the desired inequality.

3. The weak and strong Pontryagin's minimum principle. In this section we present the statements of the weak and strong Pontryagin's principles. First let us introduce some notations and definitions.

DEFINITION 3.1. We will say that problem $\left(P_{S}\right)$ is weakly stable on the right if

$$
\lim _{\delta^{\prime} \backslash \delta} \inf \left(P_{\delta^{\prime}}\right)=\inf \left(P_{\delta}\right)
$$

and weakly stable on the left if

$$
\lim _{\delta^{\prime} / \delta} \inf \left(P_{\delta^{\prime}}\right)=\inf \left(P_{\delta}\right)
$$

$\left(P_{\delta}\right)$ is said strongly stable on the right (resp. left) if there exist $\epsilon>0$ and $r>0$ such that:

$$
\inf \left(P_{\delta}\right)-\inf \left(P_{\delta^{\prime}}\right) \leq r\left(\delta^{\prime}-\delta\right) \quad \forall \delta^{\prime} \in[\delta, \delta+\epsilon]
$$


respectively

$$
\inf \left(P_{\mathcal{S}^{\prime}}\right)-\inf \left(P_{\delta}\right) \leq r\left(\delta-\delta^{\prime}\right) \quad \forall \delta^{\prime} \in[\delta-\epsilon, \delta] .
$$

If $\left(P_{\delta}\right)$ is weakly (resp. strongly) stable on the left and on the right, it will be called weakly (resp. strongly) stable.

Sufficient conditions for the weak stability were given by Casas [13] under additional regularity hypotheses on the functions $L$ and $f$. In particular, if they are continuous with respect to the third variable, $L$ is convex with respect to the same variable, $K$ is convex and closed and $\left(P_{S_{0}}\right)$ has a feasible pair $(y, u)$, then $\left(P_{\delta}\right)$ is stable on the right for every $\delta>\delta_{0}$. In spite of these results, in general it is difficult to establish the stability of a problem, mainly the strong stability. However most of problems $\left(P_{\delta}\right)$ are weak and strongly stable, more precisely:

Proposition 3.2. Let us denote by $\delta_{0}$ a real number such that $\left(P_{\delta_{0}}\right)$ has at least one feasible pair $(y, u)$. Then for every $\delta \geq \delta_{0}$, except at most a countable number of them (resp. a set of zero measure), the problem $\left(P_{\delta}\right)$ is weakly (resp. strongly) stable.

Proof. If we define $\phi:\left[\delta_{0},+\infty\right) \longrightarrow R$ by $\phi(\delta)=\inf \left(P_{\delta}\right)$, then $\phi$ is a decreasing monotone function and therefore $\phi$ is continuous (resp. differentiable) at each point except at most a countable number of them (resp. a set of zero measure). Finally it is obvious that the continuity (resp. differentiability) of $\phi$ at $\delta$ implies the weak (resp. strong) stability of $\left(P_{\delta}\right)$.

Given a number $\alpha \geq 0$, we define the Hamiltonian associated to $\left(P_{\delta}\right)$ by

$$
H_{\alpha}(x, y, u, p)=\alpha L(x, y, u)+p f(x, y, u) .
$$

If $\alpha=1$, we simply write $H$ instead of $H_{1}$. Now we can formulate the following theorems:

Theorem 3.3 (Weak Pontryagin's Principle). Let $\bar{u}$ be a solution of $\left(P_{\delta}\right)$ in $(\mathcal{K}, d)$, with $\bar{y}$ its associated state. If $\left(P_{\delta}\right)$ is weakly stable on the right, then there exist $\bar{\alpha} \geq 0, \bar{p} \in W_{0}^{1, \sigma}(\Omega)$ for every $\sigma<\frac{n}{n-1}$ and $\bar{\mu} \in M(\Omega)$ such that

$$
\left\{\begin{array}{l}
A^{\star} \bar{p}=\frac{\partial f}{\partial y}(x, \bar{y}(x), \bar{u}(x))+\bar{\alpha} \frac{\partial L}{\partial y}(x, \bar{y}(x), \bar{u}(x))+\frac{\partial g}{\partial y}(x, \bar{y}(x)) \bar{\mu} \text { in } \Omega \\
\bar{p}=0 \text { on } \Gamma
\end{array}\right.
$$

$$
\int_{\Omega}(z(x)-g(x, \bar{y}(x))) d \bar{\mu}(x) \leq 0 \quad \forall z \in C_{0}(\Omega) \text { with } z(x) \leq \delta \forall x \in \Omega,
$$

and for every $v \in K$

$$
H_{\bar{\alpha}}(x, \bar{y}(x), \bar{u}(x), \bar{p}(x)) \leq H_{\bar{\alpha}}(x, \bar{y}(x), v, \bar{p}(x)) \text { a.e. } x \in \Omega .
$$

Moreover if there exists a Lebesgue measurable set $\Omega_{0} \subset \Omega$, with $m\left(\Omega_{0}\right)=m(\Omega)$, in such a way that one of the two following conditions is satisfied 
H1) For each $y \in C_{0}(\Omega)$ and $\forall v \in K$ the set of Lebcsgue points of the functions $x \longrightarrow f(x, y(x), v)$ and $x \longrightarrow L(x, y(x), v)$ contains $\Omega_{0}$,

H2) The functions $L$ and $f$ are contimuous with respect to the third variable for every $x \in \Omega_{0}$,

then

$$
H_{\bar{\alpha}}(x, \bar{y}(x), \bar{u}(x), \bar{p}(x))=\min _{v \in K^{-}} H_{\bar{\alpha}}(x, \bar{y}(x), v, \bar{p}(x)) \text { a.e. } x \in \Omega
$$

Theorey 3.4 (Strong Pontryagin's Principle). Under the assumptions of Theorem 3.3 and assuming that $\left(P_{\delta}\right)$ is strongly stable on the right, then there exist $\bar{p} \in W_{0}^{1, \sigma}(\Omega)$ and $\bar{\mu} \in M(\Omega)$ satisfying (3.6)-(3.8), or (3.9) if the conditions $\mathbf{H 1}$ ) or H2) hold, with $\bar{\alpha}=1$.

A first version of these theorems (with stronger hypotheses) was given by Bonnans in [3] and [4]. Since we will use penalization techniques to prove these theorems, the stability on the right is the proper condition to obtain the desired result. However the Slater condition, which is a stability condition on the left, is the usual hypothesis to derive the optimality conditions (different of Pontryagin's principle) in a qualified form; see Bonnans and Casas [6]. Weak stability on the left also was the assumption in [12] to prove the convergence of the numerical approximations.

4. Hamiltonian formulation of the cost variation. In this section we generalize some results of [7] that we will use later. Let us denote by $h: \bar{\Omega} \times R \longrightarrow R$ and $\phi: R \longrightarrow R$ two functions satisfying that $\phi$ is of class $C^{1}$ and $h$ is continuous, differentiable with respect to the second variable and $\frac{\partial h}{\partial y} \in C(\bar{\Omega} \times R)$. Now we consider the functional

$$
\hat{J}(y, u)=\int_{\Omega} L(x, y(x), u(x)) d x+\phi\left(\int_{\Omega} h(x, y(x)) d x\right) .
$$

We are interested in studying this type of functionals because they play an important role in the proof of Pontryagin's principle, the second term being particularized later to some penalization of state constraints. As in the previous section

$$
H(x, y, u, p)=L(x, y, u)+p f(x, y, u) .
$$

In the first part of this section we will assume that the following regularity condition holds:

$$
\left|\frac{\partial I}{\partial y}(x, y, u)\right| \leq M_{3}(x)+\eta(|y|) \quad \forall(x, y, u) \in \Omega \times R \times K,
$$

with $M_{3} \in L^{s}(\Omega)$.

Let $u, v \in \mathcal{K}$ be two controls and $y_{u}$ and $y_{v}$ the associated states. From the mean value theorem it follows that there exist the intermediate states $\grave{y}, \hat{y}$ and $\tilde{y}$ satisfying

$$
\phi\left(\int_{\Omega} h\left(x, y_{v}(x)\right) d x\right)=\phi\left(\int_{\Omega} h\left(x, y_{u}(x)\right) d x\right)+
$$




$$
\begin{gathered}
\phi^{\prime}\left(\int_{\Omega} h(x, \check{y}(x)) d x\right) \int_{\Omega} \frac{\partial h}{\partial y}(x, \check{y}(x))\left(y_{v}(x)-y_{u}(x)\right) d x, \\
f\left(\cdot, y_{v}, v\right)=f\left(\cdot, y_{u}, v\right)+\frac{\partial f}{\partial y}(\cdot, \hat{y}, v)\left(y_{v}-y_{u}\right), \\
L\left(\cdot, y_{v}, v\right)=L\left(\cdot, y_{u}, v\right)+\frac{\partial L}{\partial y}(\cdot, \tilde{y}, v)\left(y_{v}-y_{u}\right),
\end{gathered}
$$

with $\check{y}(x), \hat{y}(x), \tilde{y}(x) \in\left[y_{u}(x), y_{v}(x)\right] \forall x \in \bar{\Omega}$. Since $y_{u}$ and $y_{v}$ are bounded, it follows that $\tilde{y}, \hat{y}$ and $\tilde{y}$ also are bounded. Now we define the intermediate adjoint state $p_{u, v}$ as solution of

$$
\left\{\begin{array}{l}
A^{\star} p_{u, v}=\frac{\partial f}{\partial y}(\cdot, \hat{y}, v) p_{u, v}+\frac{\partial L}{\partial y}(\cdot, \tilde{y}, v)+\phi^{\prime}\left(\int_{\Omega} h(x, \check{y}) d x\right) \frac{\partial h}{\partial y}(x, \check{y}) \text { in } \Omega \\
p_{u, v}=0 \text { on } \Gamma .
\end{array}\right.
$$

Note that if $u=v$, then $\tilde{y}, \hat{y}=\tilde{y}=y_{u}$ and $p_{u, v}=p_{u}$ is the adjoint state associated to $u$. Let us verify that $(4.2)$ is well posed

LEMMA 4.1. If (4.1) holds, then equation (4.2) has a unique solution $p_{u, v} \in$ $H_{0}^{1}(\Omega) \cap C^{0, \alpha}(\bar{\Omega})$ that moreover satisfies

$$
\left\|p_{u, v}\right\|_{H_{0}^{1}(\Omega)}+\left\|p_{u, v}\right\|_{C^{0, \alpha}(\bar{\Omega})} \leq C_{4} \quad \forall u, v \in \mathcal{K} .
$$

Proof. It is a straightforward consequence of Lemma 2.2 and the inequalities (2.3).

Now we have the following Hamiltonian formulation of the cost variation.

Proposition 4.2. Assume that (4.1) is satisfied and let $u, v \in \mathcal{K}$ and $p_{u, v}$ be the intermediate adjoint state associated. Then

$$
\hat{J}\left(y_{v}, v\right)=\hat{J}\left(y_{u}, u\right)+\int_{\Omega}\left[H\left(x, y_{u}(x), v(x), p_{u, v}(x)\right)-H\left(x, y_{u}(x), u(x), p_{u, v}(x)\right)\right] d x .
$$

Proof. We have

$$
\begin{gathered}
\hat{J}\left(y_{v}, v\right)-\hat{J}\left(y_{u}, u\right)=\int_{\Omega}\left[L\left(x, y_{u}(x), v(x)\right)-L\left(x, y_{u}(x), u(x)\right)\right] d x+ \\
\int_{\Omega}\left[L\left(x, y_{v}(x), v(x)\right)-L\left(x, y_{u}(x), v(x)\right)\right] d x+ \\
\\
\quad\left(\int_{\Omega} h\left(x, y_{v}(x)\right) d x\right)-\phi\left(\int_{\Omega} h\left(x, y_{u}(x)\right) d x\right) .
\end{gathered}
$$


From (4.2) we deduce

$$
\begin{gathered}
\int_{\Omega}\left[L\left(x, y_{v}(x), v(x)\right)-L\left(x, y_{u}(x), v(x)\right)\right] d x+ \\
\phi\left(\int_{\Omega} h\left(x, y_{v}(x)\right) d x\right)-\phi\left(\int_{\Omega} h\left(x, y_{u}(x)\right) d x\right)= \\
\int_{\Omega} \frac{\partial L}{\partial y}(x, \tilde{y}(x), v(x))\left(y_{v}(x)-y_{u}(x)\right) d x+ \\
\phi^{\prime}\left(\int_{\Omega} h(x, \grave{y}(x)) d x\right) \int_{\Omega} \frac{\partial h}{\partial y}(x, \grave{y}(x))\left(y_{v}(x)-y_{u}(x)\right) d x= \\
\int_{\Omega}\left[A^{\star} p_{u, v}-\frac{\partial f}{\partial y}(x, \hat{y}(x), v(x)) p_{u, v}\right]\left(y_{v}-y_{u}\right) d x= \\
\int_{\Omega} A\left(y_{v}-y_{u}\right) p_{u, v} d x-\int_{\Omega} \frac{\partial f}{\partial y}(x, \hat{y}(x), v(x)) p_{u, v}\left(y_{v}-y_{u}\right) d x= \\
\int_{\Omega}\left[f\left(x, y_{v}(x), v(x)\right)-f\left(x, y_{u}(x), u(x)\right)\right] p_{u, v} d x+ \\
\int_{\Omega}\left[f\left(x, y_{u}(x), v(x)\right)-f\left(x, y_{v}(x), v(x)\right)\right] p_{u, v} d x= \\
\left.\int_{\Omega}\left(x, y_{u}(x), v(x)\right)-f\left(x, y_{u}(x), u(x)\right)\right] p_{u, v} d x
\end{gathered}
$$

which proves the proposition.

Proposition 4.3. Assume that (4.1) holds and let $\left\{v_{k}\right\}_{k=1}^{\infty} \subset \mathcal{K}$ be a sequence converging to $u$ in the topology defined by Ekeland's distance. Then the states and the adjoint states associated $y_{k}=y_{v_{k}}$ and $p_{k}=p_{u, v_{k}}$ converge to $y_{u}$ and $p_{u}$ respectively in $H_{0}^{1}(\Omega) \cap C^{0, \alpha}(\bar{\Omega})$.

Proof. The convergence $y_{k} \rightarrow y_{u}$ follows from Theorem 2.1. The convergence of $\left\{p_{k}\right\}$ follows from the continuity of $v \in \mathcal{K} \longrightarrow p_{u, v} \in H_{0}^{1}(\Omega) \cap C^{0, \alpha}(\bar{\Omega})$ which can be proved arguing in a similar way to the proof of Theorem 2.1. $\square$

Given a point $x_{0} \in \Omega$, we will denote

$$
\omega_{k}\left(x_{0}\right)=\left\{x \in \Omega:\left\|x-x_{0}\right\| \leq 1 / k\right\}
$$

and $m_{k}\left(x_{0}\right)=m\left(\omega_{k}\left(x_{0}\right)\right)^{-1}$. We will say that a sequence $\left\{v_{k}\right\}$ in $\mathcal{K}$ is a spike perturbation of $u \in \mathcal{K}$ around $x_{0}$ associated to $v \in K$ if

$$
v_{k}(x)=\left\{\begin{array}{cl}
v & \text { if } x \in \omega_{k}\left(x_{0}\right) \\
u(x) & \text { otherwise }
\end{array}\right.
$$


Proposition 4.4. Assume that (4.1) holds and let $v_{k}$ be a spike perturbation of $u$ around $x_{0}$ associated to $v \in K$, and let $y_{k}$ be the associated state. Then for every $u \in \mathcal{K}$ there exists a set $\Omega(u, v) \subset \Omega$, with $m(\Omega(u, v))=m(\Omega)$, such that

$$
\begin{gathered}
\lim _{k \rightarrow \infty} m_{k}\left(x_{0}\right)\left[\hat{J}\left(y_{k}, v_{k}\right)-\hat{J}\left(y_{u}, u\right)\right]= \\
H\left(x_{0}, y_{u}\left(x_{0}\right), v, p_{u}\left(x_{0}\right)\right)-H\left(x_{0}, y_{u}\left(x_{0}\right), u\left(x_{0}\right), p_{u}\left(x_{0}\right)\right) \quad \forall x_{0} \in \Omega(u, v) .
\end{gathered}
$$

Proof. From Proposition 4.2 we have

$$
\hat{J}\left(y_{k}, v_{k}\right)-\hat{J}\left(y_{u}, u\right)=\int_{\omega_{k}\left(x_{0}\right)}\left[H\left(x, y_{u}(x), v, p_{k}(x)\right)-H\left(x, y_{u}(x), u(x), p_{k}(x)\right)\right] d x,
$$

where $p_{k}=p_{u, v_{k}}$ converges to $p_{u}$ in $H_{0}^{1}(\Omega) \cap C^{0, \alpha}(\bar{\Omega})$ as stated in Proposition 4.3. Then

$$
\begin{aligned}
& \hat{J}\left(y_{k}, v_{k}\right)-\hat{J}\left(y_{u}, u\right)=\int_{\omega_{k}\left(x_{0}\right)}\left[H\left(x, y_{u}(x), v, p_{u}(x)\right)-H\left(x, y_{u}(x), u(x), p_{u}(x)\right)\right] d x+ \\
& \int_{\omega_{k}\left(x_{0}\right)} f\left(x, y_{u}(x), v\right)\left(p_{k}(x)-p_{u}(x)\right) d x+\int_{\omega_{k}\left(x_{0}\right)} f\left(x, y_{u}(x), u\right)(x)\left(p_{u}(x)-p_{k}(x)\right) d x .
\end{aligned}
$$

Let $\Omega(u, v)$ the intersection of the Lebesgue points of the following mappings

$$
\begin{aligned}
& x \longrightarrow f\left(x, y_{u}(x), v\right), \\
& x \longrightarrow f\left(x, y_{u}(x), u(x)\right), \\
& x \longrightarrow H\left(x, y_{u}(x), v, p_{u}(x)\right), \\
& x \longrightarrow H\left(x, y_{u}(x), u(x), p_{u}(x)\right) .
\end{aligned}
$$

Then $m(\Omega(u, v))=m(\Omega)$. Using now the uniform convergence of $p_{k} \rightarrow p_{u}$, it follows for every $x_{0} \in \Omega(u, v)$

$$
\begin{gathered}
m_{k}\left(x_{0}\right)\left|\int_{\omega_{k}\left(x_{0}\right)} f\left(x, y_{u}(x), v\right)\left(p_{u}(x)-p_{k}(x)\right) d x\right| \leq \\
m_{k}\left(x_{0}\right) \int_{\omega_{k}\left(x_{0}\right)}\left|f\left(x, y_{u}(x), v\right)\right| d x\left\|p_{u}-p_{k}\right\|_{L^{\infty}(\Omega)} \rightarrow 0 .
\end{gathered}
$$

Analogously

$$
\begin{aligned}
& m_{k}\left(x_{0}\right)\left|\int_{\omega_{k}\left(x_{0}\right)} f\left(x, y_{u}(x), u(x)\right)\left(p_{u}(x)-p_{k}(x)\right) d x\right| \leq \\
& m_{k}\left(x_{0}\right) \int_{\omega_{k}\left(x_{0}\right)}\left|f\left(x, y_{u}(x), u(x)\right)\right| d x\left\|p_{u}-p_{k}\right\|_{L^{\infty}(\Omega)} \rightarrow 0 .
\end{aligned}
$$


Therefore we deduce

$$
\begin{gathered}
\lim _{k \rightarrow \infty} m_{k}\left(x_{0}\right)\left[\hat{J}\left(y_{k}, v_{k}\right)-\hat{J}\left(y_{u}, u\right)\right]= \\
\lim _{k \rightarrow \infty} m_{k}\left(x_{0}\right) \int_{\omega_{k}\left(x_{0}\right)}\left[H\left(x, y_{u}(x), v, p_{u}(x)\right)-H\left(x, y_{u}(x), u(x), p_{u}(x)\right)\right] d x= \\
H\left(x_{0}, y_{u}\left(x_{0}\right), v, p_{u}\left(x_{0}\right)\right)-H\left(x_{0}, y_{u}\left(x_{0}\right), u\left(x_{0}\right), p_{u}\left(x_{0}\right)\right),
\end{gathered}
$$

which concludes the proof.

The last proposition allows to deduce easily Pontryagin's principle for control problems without state constraints. In fact it is enough to suppose that $\bar{u} \in \mathcal{K}$ is a stationary point to derive a minimum principle.

DEFINITION 4.5. We say that $\bar{u}$ is a stationary point of the control problem

$$
(P)\left\{\begin{array}{l}
\min \hat{J}(y, u) \\
(y, u) \text { satisfies }(2.1) \text { and } u(x) \in K \text { a.e. } x \in \Omega
\end{array}\right.
$$

if

$$
\liminf _{d(u, \bar{u}) \rightarrow 0} \frac{\hat{J}(y, u)-\hat{J}(\bar{y}, \bar{u})}{d(u, \bar{u})} \geq 0
$$

Obviously, every local solution in $(\mathcal{K}, d)$ is a stationary point. Now we can prove the following proposition

Proposition 4.6. Let us suppose that (4.1) holds and let $\bar{u}$ be a stationary point of $(P)$. Then for every $v \in K$

$$
H(x, \bar{y}(x), \bar{u}(x), \bar{p}(x)) \leq H(x, \bar{y}(x), v, \bar{p}(x)) \text { a.e. } x \in \Omega,
$$

where $\bar{y}$ and $\bar{p}$ are the state and adjoint state associated to $\bar{u}$. Moreover, if condition H1) or H2) is verified, then $\bar{u}$ satisfies Pontryagin's principle:

$$
H(x, \bar{y}(x), \bar{u}(x), \bar{p}(x))=\min _{v \in K} H(x, \bar{y}(x), v, \bar{p}(x)) \text { a.e. } x \in \Omega .
$$

Proof. The first part of the proof is an immediate consequence of Proposition 4.4, it is enough to remark that $d\left(v_{k}, \bar{u}\right) \leq m_{k}\left(x_{0}\right)^{-1}$. To derive Pontryagin's principle under the condition H1) we use the fact that the set $\Omega(\bar{u}, v)$, defined in the proof of Proposition 4.4, contains the intersection of $\Omega_{0}$ and the set of Lebesgue points of the functions:

$$
\begin{aligned}
& x \longrightarrow f(x, \bar{y}(x), \bar{u}(x)), \\
& x \longrightarrow H(x, \bar{y}(x), \bar{u}(x), \bar{p}(x)) .
\end{aligned}
$$


Indeed the continuity of $\bar{p}$ and the condition H1) imply that $\Omega_{0}$ is a subset of the Lebesgue point set of the functions:

$$
\begin{aligned}
& x \longrightarrow f(x, \bar{y}(x), v), \\
& x \longrightarrow H(x, \bar{y}(x), v, \bar{p}(x)) .
\end{aligned}
$$

Therefore

$$
H(x, \bar{y}(x), \bar{u}(x), \bar{p}(x)) \leq H(x, \bar{y}(x), v, \bar{p}(x)) \quad \forall x \in \Omega_{0} \text { and } \forall v \in K
$$

Then

$$
H(x, \bar{y}(x), \bar{u}(x), \bar{p}(x))=\min _{v \in K} H(x, \bar{y}(x), v, \bar{p}(x)) \quad \forall x \in \Omega_{0}
$$
Then

In the case $\mathbf{H 2})$, let us take a sequence $\left\{v_{k}\right\}_{k=1}^{\infty}$ dense in $K$ and $\Omega_{1}=\cap_{k} \Omega\left(\bar{u}, v_{k}\right)$.

$$
H(x, \bar{y}(x), \bar{u}(x), \bar{p}(x)) \leq H\left(x, \bar{y}(x), v_{k}, \bar{p}(x)\right) \quad \forall x \in \Omega_{1} \text { and } \forall k \text {. }
$$

Finally the continuity of the Hamiltonian with respect to the control and the last inequality imply Pontryagin's principle in the points $x \in \Omega_{0} \cap \Omega_{1}$.

We now get rid of the regularity hypothesis (4.1).

Proposition 4.7. Let $\bar{u}$ be a local solution of $(P)$. Then the conclusions of Proposition 4.6 remain true without hypothesis (4.18).

To prove this proposition we will use Ekeland's principle:

ThEOREM 4.8 (EKELAND [15]). Let $(E, d)$ be a complete metric space, $F: E \longrightarrow$ $R \cup\{+\infty\}$ a lower semicontinuous function and let $e_{\epsilon} \in E$ satisfy

$$
F\left(e_{c}\right) \leq \inf _{e \in E} F(e)+\epsilon^{2} .
$$

Then there exists an element $\bar{e}_{\epsilon} \in E$ such that

$$
F\left(\bar{e}_{\epsilon}\right) \leq F\left(\epsilon_{\epsilon}\right), \quad d\left(\bar{e}_{\epsilon}, e_{\epsilon}\right) \leq \epsilon
$$

and

$$
F\left(\bar{\epsilon}_{\epsilon}\right) \leq F(\epsilon)+\epsilon d\left(e, \bar{e}_{\epsilon}\right) \quad \forall \epsilon \in E \text {. }
$$

Now we proceed to prove Proposition 4.7.

Proof. The idea is to regularize $L$ and to check that $\bar{u}$ is an approximate solution of the regularized problem. Using Ekeland's principle we get some optimality conditions as in Proposition 4.7 and funally we pass to the limit in these optimality conditions and get the desired result.

The regularization is as follows. By $\operatorname{Proj}_{\epsilon}$ we denote the projection onto the segment $[-1 / \epsilon,+1 / \epsilon]$, i.e.

$$
\operatorname{Proj}_{\epsilon}(t)=\max \{-1 / \epsilon, \min \{t,+1 / \epsilon\}\}
$$


We define

$$
\varphi_{c}(x, t, u)=\operatorname{Proj}_{c}\left(\frac{\partial L}{\partial y}(x, t, u)\right)
$$

and

$$
L_{\epsilon}(x, t, u)=L(x, 0, u)+\int_{0}^{t} \varphi_{c}(x, t, u) d t .
$$

We can now state the problem

$$
\left(P^{\epsilon}\right)\left\{\begin{array}{l}
\min \hat{J}_{\epsilon}(y, u)=\int_{\Omega} L_{\epsilon}(x, y(x), u(x)) d x+\phi\left(\int_{\Omega} h(x, y(x)) d x\right) \\
(y, u) \text { satisfies }(2.1), u(x) \in K \text { a.e. } x \in \Omega .
\end{array}\right.
$$

We claim that $\inf \left(P^{\epsilon}\right) \rightarrow \inf (P)$ when $\epsilon \searrow 0$. To prove this it is enough to check that

$$
\left|\hat{J}_{\epsilon}(y, u)-\hat{J}(y, u)\right| \leq r_{\epsilon} \quad \forall u \in K \text { and }(y, u) \text { satisfying }(2.1)
$$

with $r_{\epsilon} \searrow 0$ when $\epsilon \searrow 0$ and $r_{\epsilon}$ independent of $u$. Indeed, if $u_{\epsilon} \in \mathcal{K}$ satisfies that $\hat{J}_{\epsilon}\left(y_{\epsilon}, u_{\epsilon}\right) \leq \inf \left(P_{\epsilon}\right)+\varepsilon, y_{\epsilon}$ being the state associated with $u_{\epsilon}$, then by (4.4)

$$
\liminf _{\epsilon \searrow 0} \inf \left(P_{\epsilon}\right) \geq \liminf _{\epsilon \searrow 0}\left(\hat{J}_{\epsilon}\left(y_{\epsilon}, u_{\epsilon}\right)-\epsilon\right) \geq \liminf _{\epsilon}\left(\hat{J}_{\epsilon}(\bar{y}, \bar{u})-r_{\epsilon}\right)=\inf (P)
$$

and also

$$
\limsup _{\epsilon \backslash 0} \inf \left(P_{\epsilon}\right) \leq \limsup _{\epsilon \backslash 0} \hat{J}_{\epsilon}(\bar{y}, \bar{u}) \leq \limsup _{\epsilon \backslash 0}\left[\hat{J}(\bar{y}, \bar{u})+r_{\epsilon}\right]=\inf (P),
$$

which proves that $\inf \left(P_{\epsilon}\right) \rightarrow \inf (P)$, as desired. Let us now check that (4.4) holds. Indeed

$$
L(x, y, u)-L_{\epsilon}(x, y, u)=\int_{0}^{y}\left[\frac{\partial L}{\partial y}(x, t, u)-\varphi_{\epsilon}(x, t, u)\right] d t .
$$

Let $M>0$ be such that $|y(x)| \leq M$ whenever $(y, u)$ is solution of $(2.1)$ and $u \in \mathcal{K}$. Then

$$
\begin{gathered}
\left|J_{\epsilon}(y, u)-J(y, u)\right| \leq \int_{\Omega} \int_{-M}^{+M}\left|\varphi_{\epsilon}(x, t, u(x))-\frac{\partial L}{\partial y}(x, t, u(x))\right| d t d x= \\
\quad \int_{-M}^{+M} \int_{\Omega}\left|\varphi_{\epsilon}(x, t, u(x))-\frac{\partial L}{\partial y}(x, t, u(x))\right| d x d t \leq \\
\quad 2 M \int_{\Omega} \sup _{|t| \leq M, v \in K}\left|\varphi_{\epsilon}(x, t, u(x))-\frac{\partial L}{\partial y}(x, t, u(x))\right| d x .
\end{gathered}
$$


Put

$$
\Omega_{\epsilon}=\left\{x \in \Omega: \sup _{|t| \leq M, v \in K}\left|\frac{\partial L}{\partial y}(x, t, u(x))\right| \geq 1 / \epsilon\right\}
$$

As

$$
\left|\frac{\partial L}{\partial y}(x, t, u(x))\right| \leq M_{2} \in L^{1}(\Omega),
$$

it follows that $m\left(\Omega_{\varepsilon}\right) \searrow 0$ as $\epsilon \searrow 0$, and

$$
\left|\hat{J}_{\epsilon}(y, u)-\hat{J}(y, u)\right| \leq r_{\epsilon}=2 M \int_{\Omega_{e}} M_{2}(x) d x \rightarrow 0 .
$$

As a consequence of the previous results, we can get a family of real numbers $\left\{\delta_{\epsilon}\right\}_{\epsilon>0}$, with $\delta_{\epsilon} \searrow 0$ when $\epsilon \searrow 0$, such that

$$
\hat{J}_{\epsilon}(\bar{y}, \bar{u}) \leq \inf \left(P_{\epsilon}\right)+\delta_{\epsilon}^{2} .
$$

Therefore we can apply Ekeland's principle with $F(u)=\hat{J}_{\epsilon}\left(y_{u}, u\right)$ defined in the complete metric space $(\mathcal{K}, d)$, and deduce the existence of a control $u_{\epsilon} \in \mathcal{K}$ such that $d\left(\bar{u}, u_{\epsilon}\right) \leq \delta_{\epsilon}$ and

$$
\hat{J}_{\epsilon}\left(y_{\epsilon}, u_{\epsilon}\right) \leq \hat{J}_{\epsilon}\left(y_{u}, u\right)+\delta_{\epsilon} d\left(u, u_{\epsilon}\right) \quad \forall u \in \mathcal{K},
$$

where $y_{\varepsilon}$ is the state associated with $u_{\varepsilon}$. To apply Proposition 4.6 we must put the cost given by the right hand side of inequality (4.5) into the framework of this proposition. For it we introduce the function $\chi_{\epsilon}: \Omega \times K \longrightarrow R$ by

$$
\chi_{\epsilon}(x, v)= \begin{cases}0 & \text { if } v=u_{\epsilon}(x) \\ 1 & \text { otherwise }\end{cases}
$$

Then $\left(y_{\epsilon}, u_{\epsilon}\right)$ is the solution of the problem

$$
\left(Q^{c}\right)\left\{\begin{array}{l}
\min \hat{J}_{\epsilon}(y, u)=\hat{J}_{\epsilon}(y, u)+\delta_{\epsilon} \int_{\Omega} \chi_{\epsilon}(x, u(x)) \\
(y, u) \text { satisfies }(2.1), u(x) \in K \text { a.e. } x \in \Omega .
\end{array}\right.
$$

Then Proposition 4.6 implies that for every $v \in K$

$$
H^{\epsilon}\left(x, y_{\epsilon}(x), u_{\epsilon}(x), p_{\epsilon}(x)\right) \leq H^{\epsilon}\left(x, y_{\epsilon}(x), v, p_{\epsilon}(x)\right)+\delta_{\epsilon} \quad \text { a.e. } \quad x \in \Omega
$$

where $p_{\epsilon}$ is the adjoint state

$$
\left\{\begin{array}{l}
A^{\star} p_{\epsilon}=\frac{\partial f}{\partial y}\left(x, y_{\epsilon}, u_{\epsilon}\right) p_{\epsilon}+\frac{\partial L_{\epsilon}}{\partial y}\left(x, y_{\epsilon}, u_{\epsilon}\right) \text { in } \Omega \\
p_{\epsilon}=0 \text { on } \Gamma
\end{array}\right.
$$


and

$$
H^{\iota}(x, y, u, p)=L_{\epsilon}(x, y, u)+p f(x, y, u)+\delta_{\epsilon} \chi_{\epsilon}(x, u) .
$$

From Theorem 2.1 it follows that $y_{\epsilon} \rightarrow \bar{y}$ in $H_{0}^{1}(\Omega) \cap C^{0 . \alpha}(\bar{\Omega})$. 'Then, thanks to hypothesis (2.3) and the definition of $L_{\epsilon}$, we have

$$
\begin{aligned}
& f\left(x, y_{\epsilon}, u_{\epsilon}\right) \rightarrow f(x, \bar{y}, \bar{u}) \quad \text { and } \quad \frac{\partial f}{\partial y}\left(x, y_{\epsilon}, u_{\epsilon}\right) \rightarrow \frac{\partial f}{\partial y}(x, \bar{y}, \bar{u}) \quad \text { in } L^{s}(\Omega), \\
& L_{\epsilon}\left(x, y_{\epsilon}, u_{\epsilon}\right) \rightarrow L(x, \bar{y}, \bar{u}) \quad \text { and } \quad \frac{\partial L_{\epsilon}}{\partial y}\left(x, y_{\epsilon}, u_{\epsilon}\right) \rightarrow \frac{\partial L}{\partial y}(x, \bar{y}, \bar{u}) \quad \text { in } L^{1}(\Omega) .
\end{aligned}
$$

With the aid of these relations and Lemma 2.4 we can pass to the limit in (4.6) and (4.7) and to deduce the first conclusion of Proposition 4.6. To prove the second conclusion, i.e. the Pontryagin's principles. we argue as follows. Under condition H2), the argument used in the proof of Proposition 4.6 can be repeated here without modifications. If condition $\mathbf{H 1}$ ) is satisfied, then. thanks to Proposition 4.6, we can take a sequence $c_{j} \searrow 0$ and $\delta_{j}=\delta_{\epsilon}, \searrow 0$ such that

$$
H^{\epsilon_{j}}\left(x, y_{\epsilon_{j}}(x), u_{\epsilon_{j}}(x), p_{\epsilon_{j}}(x)\right) \leq \min _{v \in K} H^{\epsilon,}\left(x, y_{\epsilon_{j}}(x), v, p_{\epsilon_{j}}(x)\right)+\delta_{j} \quad \text { a.e. } x \in \Omega_{j},
$$

with $m\left(\Omega_{j}\right)=m(\Omega)$. Now we pass to the limit and get

$$
H(x, \bar{y}(x), \bar{u}(x), \bar{p}(x))=\min _{v \in K^{.}} H(x, \bar{y}(x), v, \bar{p}(x)) \quad \text { a.e. } \quad x \in \tilde{\Omega}=\bigcap_{j=1}^{\infty} \Omega_{j},
$$

which concludes the proof because $m(\tilde{\Omega})=m(\Omega)$.

THEOREM 4.9. The statement of Proposition 4.6 is still valid without hypothesis (4.1).

Proof. From the definition of stationary point we deduce that for every $\epsilon>0$ there exists $r_{\varepsilon}>0$ such that

$$
\frac{\hat{J}(y, u)-\hat{J}(\bar{y}, \bar{u})}{d(u, \bar{u})} \geq-\epsilon \quad \forall u \in B_{r_{e}}(\bar{u})
$$

where $B_{r_{e}}(\bar{u})$ is the open ball of $(\mathcal{K}, d)$ of radius $r_{\epsilon}$ and center at $\bar{u}$. Hence

$$
\hat{J}(\bar{y}, \bar{u}) \leq \hat{J}(y, u)+\epsilon \int_{\Omega} \chi(x, u(x)) d x \quad \forall u \in B_{r_{\epsilon}}(\bar{u})
$$

with

$$
\chi(x, v)= \begin{cases}0 & \text { if } v=\bar{u}(x) \\ 1 & \text { otherwise }\end{cases}
$$

Then it is enough to apply Proposition 4.7 to the problem

$$
\left\{\begin{array}{l}
\min \hat{J}(y, u)+\epsilon \int_{\Omega} \chi(x, u(x)) d x \\
u \in B_{r_{\epsilon}}(\bar{u}), u(x) \in K
\end{array}\right.
$$


and pass to the limit when $\epsilon \searrow 0$, to deduce the desired result.

The hypotheses made about $K, L$ and $f$ do not allow to assure the existence of a solution of control problem $(P)$. Here we will prove a principle of Pontryagin's type for $\epsilon$-solutions.

Definition 4.10. A control $u \in \mathcal{K}$ is called an $\epsilon$-solution of $(P)$ if $\hat{J}\left(y_{u}, u\right) \leq \inf (P)+\epsilon$.

THEOREM 4.11. For every $\epsilon>0$ there exists at least one $\epsilon^{2}$-solution of $(P)$ in $\mathcal{K}$. Furthermore for every $\epsilon^{2}$-solution of $(P), \bar{u}_{\epsilon}$, there exists another $\epsilon^{2}$-solution $u_{\epsilon}$ such that $d\left(u_{\epsilon}, \bar{u}_{\epsilon}\right) \leq \epsilon$ and for every $v \in K$

$$
H\left(x, y_{\epsilon}(x), u_{\epsilon}(x), p_{\epsilon}(x)\right) \leq H\left(x, y_{\epsilon}(x), v, p_{\epsilon}(x)\right)+\epsilon \text { a.e. } x \in \Omega,
$$

where $y_{\epsilon}=y_{u_{\epsilon}}$ and $p_{\epsilon}=p_{u_{\epsilon}}$. Moreover, if there exists a Lebesgue measurable set $\Omega_{0} \subset \Omega$, with $m\left(\Omega_{0}\right)=m(\Omega)$, in such a way that $\mathbf{H 1}$ ) or $\mathbf{H 2 )}$ holds, then $\bar{u}$ satisfies Pontryagin's principle:

$$
H\left(x, y_{\epsilon}(x), u_{\epsilon}(x), p_{\epsilon}(x)\right)=\min _{v \in K} H\left(x, y_{\epsilon}(x), v, p_{\epsilon}(x)\right)+\epsilon \text { a.e. } x \in \Omega .
$$

Proof. Thanks to the hypothesis (2.3), we have that $\inf (P) \in R$. Therefore there exists at least one $\epsilon^{2}$-solution of $(P)$. Let $\bar{u}_{\epsilon}$ be one of them. Then we can apply Theorem 4.8, with $F(u)=\hat{J}\left(y_{u}, u\right)$ defined in the metric space $(\mathcal{K}, d)$, and deduce the existence of a control $u_{\epsilon} \in \mathcal{K}, \epsilon^{2}$-solution of $(P)$, such that $d\left(u_{\epsilon}, \bar{u}_{\epsilon}\right) \leq \epsilon$ and

$$
\hat{J}\left(y_{u_{e}}, u_{\epsilon}\right) \leq \hat{J}\left(y_{u}, u\right)+\epsilon d\left(u, u_{\epsilon}\right) \quad \forall u \in \mathcal{K} .
$$

Now we put the cost into the framework of Proposition 4.7, using the function $\chi_{\epsilon}$ as in its proof, and apply it to get the result. $\square$

5. Proof of the weak minimum principle. Let $\bar{u}$ be a solution of $\left(P_{\delta}\right)$ and $\bar{y}$ its associated state. For every $\gamma>0$ we define the problems

$$
\left(Q_{\gamma}\right)\left\{\begin{array}{l}
\min J_{\gamma}(y, u)=\int_{\Omega}\left[L(x, y(x), u(x))+\frac{1}{2 \gamma}\left((g(x, y(x))-\delta)^{+}\right)^{2}\right] d x \\
(y, u) \text { satisfies }(2.1) \text { and } u(x) \in K \text { a.e. } x \in \Omega .
\end{array}\right.
$$

The first issue to remark is the following

Proposition 5.1. Let $\left(P_{\hat{j}}\right)$ be weakly stable on the right. Then

$$
\lim _{\gamma \backslash 0} \inf \left(Q_{\gamma}\right)=\inf \left(P_{S}\right)
$$

Proof. Let $\left\{u_{\gamma}\right\}$ be a family of $\gamma$-solutions of problems $\left(Q_{\gamma}\right)$ and $\left\{y_{\gamma}\right\}$ the associated states:

$$
J\left(y_{\gamma}, u_{\gamma}\right) \leq \inf \left(Q_{\gamma}\right)+\gamma
$$


¿lrom the definition of $\left(Q_{\gamma}\right)$ it follows that $\left(g\left(x, y_{\gamma}(x)\right)-\delta\right)^{+} \rightarrow 0$ in $L^{2}(\Omega)$, which, together with (2.4) and the compactness of the inclusion $H_{0}^{1}(\Omega) \cap C^{0, \alpha}(\bar{\Omega}) \subset C_{0}(\Omega)$, implies the convergence $\left(g\left(x, y_{\gamma}(x)\right)-\delta\right)^{+} \rightarrow 0$ in $C_{0}(\Omega)$. Therefore

$$
\delta_{\gamma}=\left\|\left(g\left(x, y_{\gamma}(x)\right)-\delta\right)^{+}\right\|_{L^{\infty}(\Omega)}+\delta \rightarrow \delta \text { if } \gamma \searrow 0 .
$$

As $\left(y_{\gamma}, u_{\gamma}\right)$ is feasible pair for $\left(P_{\delta_{\gamma}}\right)$ we deduce that

$$
\inf \left(P_{\delta_{\gamma}}\right) \leq J\left(y_{\gamma}, u_{\gamma}\right) \leq \inf \left(Q_{\gamma}\right)+\gamma
$$

Then, using the weak stability of $\left(P_{\delta}\right)$ on the right, we obtain

$$
\inf \left(P_{\delta}\right)=\lim _{\gamma \backslash 0} \inf \left(P_{\delta_{\gamma}}\right) \leq \lim _{\gamma \searrow 0}\left\{\inf \left(Q_{\gamma}\right)+\gamma\right\}=\lim _{\gamma \searrow 0} \inf \left(Q_{\gamma}\right) \leq \inf \left(P_{\delta}\right)
$$

with the last inequality due to the fact that $\left(y_{u}, u\right)$ is feasible for $\left(Q_{\gamma}\right)$ whenever it is feasible for $\left(P_{\delta}\right)$, with the same cost.

Proof of Theorem 3.3 Thanks to Proposition 5.1 we deduce that $\bar{u}$ is a $\epsilon_{\gamma}^{2}$-solution of $\left(Q_{\gamma}\right)$, with $\epsilon_{\gamma} \searrow 0$ when $\gamma \searrow 0$. Applying Theorem 4.11 we obtain the existence of a control $u_{\gamma} \in \mathcal{K}, \epsilon_{\gamma}^{2}$-solution of $\left(Q_{\gamma}\right)$, with $d\left(u_{\gamma}, \bar{u}\right) \leq \epsilon_{\gamma}$ and such that for every $v \in K$

$$
H\left(x, y_{\gamma}(x), u_{\gamma}(x), p_{\gamma}(x)\right) \leq H\left(x, y_{\gamma}(x), v, p_{\gamma}(x)\right)+\epsilon_{\gamma} \text { a.e. } x \in \Omega,
$$

where $y_{\gamma}=y_{u_{\gamma}}$ and $p_{\gamma}$ is the adjoint state:

$$
\left\{\begin{array}{l}
A^{\star} p_{\gamma}=\frac{\partial f}{\partial y}\left(x, y_{\gamma}, u_{\gamma}\right) p_{\gamma}+\frac{\partial L}{\partial y}\left(x, y_{\gamma}, u_{\gamma}\right)+\frac{1}{\gamma}\left(g\left(x, y_{\gamma}\right)-\delta\right)^{+} \frac{\partial g}{\partial y}\left(x, y_{\gamma}\right) \text { in } \Omega \\
p_{\gamma}=0 \text { on } \Gamma
\end{array}\right.
$$

Defining

$$
\begin{aligned}
& \bar{\alpha}_{\gamma}=\left(1+\left\|\frac{1}{\gamma}\left(g\left(x, y_{\gamma}\right)-\delta\right)^{+}\right\|_{M(\Omega)}\right)^{-1} \\
& \bar{\mu}_{\gamma}=\frac{\alpha_{\gamma}}{\gamma}\left(g\left(x, y_{\gamma}\right)-\delta\right)^{+}, \text {and } \bar{p}_{\gamma}=\bar{\alpha}_{\gamma} p_{\gamma}
\end{aligned}
$$

we get

$$
\left\{\begin{array}{l}
A^{\star} \bar{p}_{\gamma}=\frac{\partial f}{\partial y}\left(x, y_{\gamma}, u_{\gamma}\right) \bar{p}_{\gamma}+\bar{\alpha}_{\gamma} \frac{\partial L}{\partial y}\left(x, y_{\gamma}, u_{\gamma}\right)+\bar{\mu}_{\gamma} \frac{\partial g}{\partial y}\left(x, y_{\gamma}\right) \text { in } \Omega \\
\bar{p}_{\gamma}=0 \text { on } \Gamma
\end{array}\right.
$$

and for every $v \in K$

$$
H_{\bar{\alpha}_{\gamma}}\left(x, y_{\gamma}(x), u_{\gamma}(x), \bar{p}_{\gamma}(x)\right) \leq H_{\bar{\alpha}_{\gamma}}\left(x, y_{\gamma}(x), v, \bar{p}_{\gamma}(x)\right)+\epsilon_{\gamma} \text { a.e. } x \in \Omega .
$$


If $\gamma \searrow 0$, then $d\left(u_{\gamma}, \bar{u}\right) \leq \epsilon_{\gamma} \rightarrow 0$, therefore from Theorem 2.1 we get $y_{\gamma} \rightarrow \bar{y}$ in $H_{0}^{1}(\Omega) \cap C^{0, \alpha}(\bar{\Omega})$. Using now $(2.3)$, we deduce the convergences

$$
\begin{array}{rlrl}
f\left(x, y_{\gamma}, u_{\gamma}\right) & \rightarrow f(x, \bar{y}, \bar{u}), & \frac{\partial f}{\partial y}\left(x, y_{\gamma}, u_{\gamma}\right) \rightarrow \frac{\partial f}{\partial y}(x, \bar{y}, \bar{u}) \text { in } L^{s}(\Omega), \\
L\left(x, y_{\gamma}, u_{\gamma}\right) \rightarrow L(x, \bar{y}, \bar{u}), & \frac{\partial L}{\partial y}\left(x, y_{\gamma}, u_{\gamma}\right) \rightarrow \frac{\partial L}{\partial y}(x, \bar{y}, \bar{u}) \text { in } L^{s}(\Omega) .
\end{array}
$$

Applying Lemma 2.4, we obtain the following estimation for $\bar{p}_{\gamma}$

$$
\left\|\bar{p}_{\gamma}\right\|_{W_{0}^{1, \sigma}(\Omega)} \leq M\left\|\bar{\alpha}_{\gamma} \frac{\partial L}{\partial y}\left(x, y_{\gamma}, u_{\gamma}\right)+\bar{\mu}_{\gamma} \frac{\partial g}{\partial y}\left(x, y_{\gamma}(x)\right)\right\|_{M(\Omega)} \leq M^{\prime}<+\infty
$$

for every $\sigma<n /(n-1)$. Therefore, remembering (5.1), we can extract subsequences, denoted in the same way, such that $\bar{\mu}_{\gamma} \rightarrow \bar{\mu}$ in $M(\Omega)$ *weakly and $\bar{p}_{\gamma} \rightarrow \bar{p}$ weakly in $W_{0}^{1, \sigma}(\Omega)$. From the Rellich's theorem (Adams [1]) it follows $\bar{p}_{\gamma} \rightarrow \bar{p}$ strongly in $L^{q}(\Omega)$ for each $q<n /(n-2)$. Then

$$
\frac{\partial f}{\partial y}\left(x, y_{\gamma}, u_{\gamma}\right) \bar{p}_{\gamma} \rightarrow \frac{\partial f}{\partial y}(x, \bar{y}, \bar{u}) \bar{p} \text { in } L^{1}(\Omega) .
$$

We can pass to the limit in (5.2) and derive (3.6). Relation (3.8) follows from (5.3). Relation (3.7) is obtained as follows: for every $z \in C_{0}(\Omega)$ with $z(x) \leq \delta$ for all $x \in \Omega$

$$
\int_{\Omega}(z(x)-g(x, \bar{y}(x))) d \bar{\mu}(x)=\lim _{\gamma \rightarrow 0} \frac{\bar{\alpha}_{\gamma}}{\gamma} \int_{\Omega}\left(z(x)-g\left(x, y_{\gamma}(x)\right)\right)\left(g\left(x, y_{\gamma}(x)\right)-\delta\right)^{+} d x \leq 0 .
$$

It follows that

$$
<\bar{\mu}, g(\cdot, \bar{y})>=\max \left\{\left\langle\bar{\mu}, z>: z \in C_{0}(\Omega), z(x) \leq \delta\right\}\right.
$$

hence $\bar{\mu}$ is nonnegative and the value of the $\max$ is $\delta\|\bar{\mu}\|_{M(\Omega)}$.

To obtain (3.5) it is enough to remember (5.1) and remark that

$$
\|\bar{\mu}\|_{M(\Omega)}=\frac{1}{\delta}<\bar{\mu}, g(\cdot, \bar{y})>=\lim _{\gamma \rightarrow 0} \frac{1}{\delta}<\bar{\mu}_{\gamma}, g\left(\cdot, y_{\gamma}\right)>=\lim _{\gamma \rightarrow 0}\left\|\bar{\mu}_{\gamma}\right\|_{M(\Omega)} .
$$

Finally we must prove (3.9). If we assume that $\mathbf{H 1}$ ) is satisfied, then, thanks to Theorem $4.11,(5.3)$ can be written

$$
H_{\bar{\alpha}_{\gamma}}\left(x, y_{\gamma}(x), u_{\gamma}(x), \bar{p}_{\gamma}(x)\right)=\min _{v \in K} H_{\bar{\alpha}_{\gamma}}\left(x, y_{\gamma}(x), v, \bar{p}_{\gamma}(x)\right)+\epsilon_{\gamma} \text { a.e. } x \in \Omega .
$$

Taking a subsequence $\left\{\gamma_{j}\right\}_{j=1}^{\infty}$, we pass to the limit as above and get (3.9).

If H2) is satisfied, we can argue as in the proof of Theorem 4.9 to conclude (3.9). $\square$ 
6. Proof of the strong minimum principle. In this section we establish the existence of a certain link between the stability of the cost with respect to small perturbations of the feasible state set and the viability of the exact penalization procedure of the state constraints. In the context of the abstract optimization Burke [10], generalizing an idea of Clarke [14], proved an equivalence result between stability and exact penalization. Since we are assuming the hypotheses of Theorem 3.4 , we have that $\left(P_{\delta}\right)$ is strongly stable on the right and $(\bar{y}, \bar{u})$ is a solution of this problem. Now we consider the exact penalization of state constraints:

Proposition 6.1. If $r>0$ satisfies (3.3), then $\bar{u}$ is a local solution in $(\mathcal{K}, d)$ of the penalized control problem

$$
\left\{\begin{array}{l}
\min J_{r}(u)=\int_{\Omega} L\left(x, y_{u}(x), u(x)\right) d x+r\left\|\left(g\left(x, y_{u}\right)-\delta\right)^{+}\right\|_{\infty} \\
u \in \mathcal{K} .
\end{array}\right.
$$

Proof. From (3.3) it follows

$$
\inf \left(P_{\delta}\right)=\inf \left\{J\left(y_{u}, u\right)+r\left(\delta^{\prime}-\delta\right): u \in \mathcal{K}, g\left(x, y_{u}(x)\right) \leq \delta^{\prime}, \delta^{\prime} \in[\delta, \delta+\epsilon]\right\} .
$$

Minimizing first with respect to $\delta^{\prime}$ for fixed $u$ we find

$$
\inf \left(P_{\mathcal{S}}\right)=\inf \left\{J\left(y_{u}, u\right)+r\left\|\left(g\left(x, y_{u}\right)-\delta\right)^{+}\right\|_{\infty}: u \in \mathcal{K}, g\left(x, y_{u}(x)\right) \leq \delta+\epsilon\right\}
$$

Since the mapping $u \in(\mathcal{K}, d) \longrightarrow y_{u} \in C_{0}(\Omega)$ is continuous, we deduce the existence of a ball $B_{\lambda}(\bar{u}), \lambda>0$, such that

$$
\left\|g\left(x, y_{u}\right)\right\|_{\infty}<\delta+\epsilon \quad \forall u \in B_{\lambda}(\bar{u})
$$

which, together with the previous identity, proves that $\bar{u}$ is a local solution of the penalized control problem.

Take $\lambda>0$ as in the proof of the previous proposition and $r>0$ verifying (3.3). We introduce the problem

$$
\left(Q_{\tau}\right)\left\{\begin{array}{l}
\left.\min J_{r}(u)=\int_{\Omega} L\left(x, y_{u}(x), u(x)\right) d x+r \|\left(g\left(x, y_{u}\right)-\delta\right)^{+}\right) \|_{\infty} \\
u \in B_{\lambda}(\bar{u}) .
\end{array}\right.
$$

Then $\bar{u}$ is a solution of this problem. We passed from a state-constrained control problem to another control problem without state constraints. The difficulty in this new problem is that the penalization term is not differentiable. To overcome this difficulty we define

$$
\left.J_{r, q}(u)=\int_{\Omega} L\left(x, y_{u}(x), u(x)\right) d x+r\left(q^{-q}+\int_{\Omega}\left[\left(g\left(x, y_{u}(x)\right)-\delta\right)^{+}\right]^{q}\right) d x\right)^{1 / q}
$$

and

$$
\left(Q_{r, q}\right)\left\{\begin{array}{l}
\min J_{r, q}(u) \\
u \in B_{\lambda}(\bar{u})
\end{array}\right.
$$


with $q>1$. Note that $\left(Q_{r, q}\right)$ has a differentiable cost and moreover it represents an approximation of $\left(Q_{\tau}\right)$ :

PROPOSITION 6.2. The following identity holds

$$
\inf \left(Q_{r}\right)=\lim _{q \rightarrow \infty} \inf \left(Q_{r, q}\right)
$$

Proof. From the convergence $\|z\|_{L^{q}(\Omega)} \rightarrow\|z\|_{\infty}$ for every $z \in L^{\infty}(\Omega)$ and the inequalities

$$
\begin{gathered}
\left.\left\|\left(g\left(x, y_{u}\right)-\delta\right)^{+}\right\|_{L^{q}(\Omega)} \leq\left(q^{-q}+\int_{\Omega}\left[\left(g\left(x, y_{u}(x)\right)-\delta\right)^{+}\right]^{q}\right) d x\right)^{1 / q} \leq \\
\frac{1}{q}+\left\|\left(g\left(x, y_{u}\right)-\delta\right)^{+}\right\|_{L^{q}(\Omega)}
\end{gathered}
$$

we deduce that $J_{r, q}(u) \rightarrow J_{r}(u)$ when $q \rightarrow+\infty$. Therefore for every $u \in B_{\lambda}(\bar{u})$

$$
\limsup _{q \rightarrow+\infty} \inf \left(Q_{r, q}\right) \leq \limsup _{q \rightarrow+\infty} J_{r, q}(u)=J_{r}(u),
$$

hence

$$
\limsup _{q \rightarrow+\infty} \inf \left(Q_{r, q}\right) \leq \inf \left(Q_{r}\right)
$$

Now we prove the converse inequality. Let us take $\epsilon>0$ arbitrary and let $C_{1}>0$ be the constant given in Theorem 2.1. Since $g: \bar{\Omega} \times R \longrightarrow R$ is continuous, it follows the existence of a constant $\rho \in(0, \epsilon)$ such that $\forall x, x^{\prime} \in \bar{\Omega}$

$$
\left|g(x, t)-g\left(x^{\prime}, t\right)\right|<\epsilon \quad \text { if } \quad\left|x-x^{\prime}\right| \leq \rho \text { and }|t| \leq C_{1} .
$$

Moreover we assume $\rho$ small enough in such a way that $m(\{x:|x| \leq \rho\})<1$. Now we define $\Omega_{\rho}\left(x_{0}\right)=\Omega \cap B_{\rho}\left(x_{0}\right)$. Since the boundary of $\Gamma$ is Lipschitz, there exists a number $\beta \in(0,1)$ verifying

$$
m\left(\Omega_{p}\left(x_{0}\right)\right) \geq \beta m(\{x:|x| \leq \rho\}) \quad \forall x_{0} \in \bar{\Omega}
$$

On the other hand, from the continuity of $\frac{\partial g}{\partial y}$ we deduce the existence of another constant $M>0$ such that

$$
|g(x, t)|+\left|\frac{\partial g}{\partial y}(x, t)\right| \leq M \quad \forall(x, t) \in \bar{\Omega} \times\left[-C_{1},+C_{1}\right] .
$$

Pick $u \in B_{\lambda}(\pi)$. If $\left\|\left(g\left(x, y_{u}\right)-\delta\right)^{+}\right\|_{\infty}=0$, then $J_{r, q}(u)=J_{r}(u)$. Let us suppose that $\left\|\left(g\left(x, y_{u}\right)-\delta\right)^{+}\right\|_{\infty}>0$ and take $x_{0} \in \bar{\Omega}$ verifying

$$
\left(g\left(x_{0} \cdot y_{u}\left(x_{0}\right)\right)-\delta\right)^{+}=\left\|\left(g\left(x, y_{u}\right)-\delta\right)^{+}\right\|_{\infty} .
$$


Then for cach $x \in \Omega_{\rho}\left(x_{0}\right)$ we get with the aid of $(2.4),(6.2)$ and $(6.3)$

$$
\begin{gathered}
\left|g\left(x, y_{u}(x)\right)-g\left(x_{0}, y_{u}\left(x_{0}\right)\right)\right| \leq \\
\left|g\left(x, y_{u}(x)\right)-g\left(x_{0}, y_{u}(x)\right)\right|+\left|g\left(x_{0}, y_{u}(x)\right)-g\left(x_{0}, y_{u}\left(x_{0}\right)\right)\right| \leq \\
\epsilon+M\left|y_{u}(x)-y_{u}\left(x_{0}\right)\right| \leq \epsilon+M C_{1} \rho^{\alpha} \leq M^{\prime} \epsilon,
\end{gathered}
$$

hence

$$
\left(g\left(x, y_{u}(x)\right)-\delta\right)^{+} \geq\left(g\left(x_{0}, y_{u}\left(x_{0}\right)\right)-\delta-M^{\prime} \epsilon\right)^{+} \forall x \in \Omega_{\rho}\left(x_{0}\right) .
$$

Therefore, we obtain

$$
\begin{gathered}
\left.\left\|\left(g\left(x, y_{u}\right)-\delta\right)^{+}\right\|_{L^{q}(\Omega)} \geq\left(\int_{\Omega_{\rho}\left(x_{0}\right)}\left[g\left(x, y_{u}(x)\right)-\delta\right)^{+}\right]^{q} d x\right)^{1 / q} \geq \\
m\left(\Omega_{\rho}\left(x_{0}\right)\right)^{1 / q}\left(g\left(x_{0}, y_{u}\left(x_{0}\right)\right)-\delta-M^{\prime} \epsilon\right)^{+} \geq\left\|\left(g\left(x, y_{u}\right)-\delta\right)^{+}\right\|_{\infty}+ \\
\left(m\left(\Omega_{\rho}\left(x_{0}\right)\right)^{1 / q}-1\right)\left\|\left(g\left(x, y_{u}\right)-\delta\right)^{+}\right\|_{\infty}-M^{\prime} \epsilon m\left(\Omega_{\rho}\left(x_{0}\right)\right)^{1 / q} \geq \\
\left\|\left(g\left(x, y_{u}\right)-\delta\right)^{+}\right\|_{\infty}+M\left(m\left(\Omega_{\rho}\left(x_{0}\right)\right)^{1 / q}-1\right)-M^{\prime} \epsilon .
\end{gathered}
$$

Choosing $q_{\epsilon}>1$ such that

$$
1-m\left(\Omega_{\rho}\left(x_{0}\right)\right)^{1 / q} \leq 1-[\beta m(\{x:|x| \leq \rho\})]^{1 / q}<\epsilon \quad \forall q>q_{\iota},
$$

it follows

$$
\left\|\left(g\left(x, y_{u}\right)-\delta\right)^{+}\right\|_{L^{q}(\Omega)} \geq\left\|\left(g\left(x, y_{u}\right)-\delta\right)^{+}\right\|_{\infty}-\left(M+M^{\prime}\right) \epsilon \quad \forall q>q_{\epsilon} .
$$

We have proved that

$$
J_{r, q}(u) \geq J_{r}(u)-\left(M+M^{\prime}\right) \epsilon
$$

for each $u \in B_{\lambda}(\bar{u})$ and all $q \geq q_{\epsilon}$, hence

$$
\liminf _{q \rightarrow+\infty} \inf \left(Q_{r, q}\right) \geq \inf \left(Q_{r}\right)-\left(M+M^{\prime}\right) \epsilon
$$

for $\epsilon>0$ arbitrary, consequently

$$
\liminf _{q \rightarrow+\infty} \inf \left(Q_{r, q}\right) \geq \inf \left(Q_{r}\right) .
$$

So the proposition follows from (6.1) and (6.4). 
Proof of Thcorem 3.4 Thanks to Proposition 6.2 we deduce that $\bar{u}$ is an $\epsilon_{q}^{2}$-solution of $\left(Q_{r, q}\right)$, with $\epsilon_{q} \rightarrow 0$ as $q \rightarrow \infty$. Then Theorem 4.11 states the existence of a control $u_{q} \in \mathcal{K}$, with $d\left(u_{q}, \bar{u}\right) \leq \epsilon_{q}$, satisfying for every $v \in K$

$$
H\left(x, y_{q}(x), u_{q}(x), p_{q}(x)\right) \leq H\left(x, y_{q}(x), v, p_{q}(x)\right)+\epsilon_{q} \text { a.e. } x \in \Omega,
$$

where $y_{q}=y_{u_{q}}$ and $p_{q}$ is the adjoint state:

$$
\left\{\begin{array}{l}
A^{\star} p_{q}=\frac{\partial f}{\partial y}\left(x, y_{q}, u_{q}\right) p_{q}+\frac{\partial L}{\partial y}\left(x, y_{q}, u_{q}\right)+\mu_{q} \frac{\partial g}{\partial y}\left(x, y_{q}\right) \text { in } \Omega \\
p_{q}=0 \text { on } \Gamma
\end{array}\right.
$$

with

$$
\mu_{q}=r\left(q^{-q}+\int_{\Omega}\left[\left(g\left(x, y_{q}(x)\right)-\delta\right)^{+}\right]^{q} d x\right)^{1 / q-1}\left[\left(g\left(x, y_{q}\right)-\delta\right)^{+}\right]^{q-1}
$$

Now we must pass to the limit. From $d\left(u_{q}, \bar{u}\right) \leq \epsilon_{q} \rightarrow 0$ and Theorem 2.1 we obtain that $y_{q} \rightarrow \bar{y}$ in $H_{0}^{1}(\Omega) \cap C^{0, \alpha}(\bar{\Omega})$. On the other hand, from the definition of $\mu_{q}$ we get

$$
\begin{gathered}
\left\|\mu_{q}\right\|_{M(\Omega)}=\left\|\mu_{q}\right\|_{L^{1}(\Omega)} \leq r\left(\int _ { \Omega } [ ( g ( x , y _ { q } ( x ) ) ^ { + } ] ^ { q } d x ) ^ { 1 / q - 1 } \int _ { \Omega } \left[\left(g\left(x, y_{q}(x)\right)^{+}\right]^{q-1} d x=\right.\right. \\
r\left\|\left(g\left(x, y_{q}\right)-\delta\right)^{+}\right\|_{L^{q}(\Omega)}^{1-q}\left\|\left(g\left(x, y_{q}\right)-\delta\right)^{+}\right\|_{L^{q-1}(\Omega)}^{q-1} .
\end{gathered}
$$

Applying Hölder's inequality with exponents $q /(q-1)$ and $q$ it follows

$$
\|z\|_{L^{q-1}(\Omega)} \leq m(\Omega)^{1 / q}\|z\|_{L^{q}(\Omega)} \forall z \in L^{q}(\Omega)
$$

which together with the previous relation leads to

$$
\begin{gathered}
\left\|\mu_{q}\right\|_{M(\Omega)} \leq m(\Omega)^{1 / q} r\left\|\left(g\left(x, y_{q}\right)-\delta\right)^{+}\right\|_{L^{q}(\Omega)} \leq \\
m(\Omega)^{2 / q} r\left\|\left(g\left(x, y_{q}\right)-\delta\right)^{+}\right\|_{\infty} \leq M<+\infty \quad \forall q>1 .
\end{gathered}
$$

As in the proof of Theorem 3.3, the boundedness of $\left\{\mu_{q}\right\}_{q \geq 1}$, the convergence of $\left\{\left(y_{q}, u_{q}\right)\right\}_{q \geq 1}$ and assumptions (2.3) imply the boundedness of $\left\{p_{q}\right\}_{q \geq 1}$ in $W_{0}^{1, \sigma}(\Omega)$ for every $\sigma<n /(n-1)$. Therefore we can extract subsequences $\left\{p_{q_{k}}\right\}$ and $\left\{\mu_{q_{k}}\right\}$, with $q_{k} \rightarrow+\infty$, converging to $\bar{p}$ and $\bar{\mu}$ in $W_{0}^{1, \sigma}(\Omega)$ weakly and $M(\Omega)^{*}$-weakly respectively. Now it is easy to pass to the limit in (6.5) and (6.6) and to obtain (3.8) and (3.6). As in the proof of Theorem 3.3 we derive (3.7) from the definition of $\mu_{q}$.

Finally; as stated in Theorem 4.11, under the conditions $\mathbf{H 1}$ ) or H2) given in Theorem 3.3, the relation $(6.5)$ becomes

$$
H\left(x, y_{q}(x), u_{q}(x), p_{q}(x)\right)=\min _{v \in K} H\left(x, y_{q}(x), v, p_{q}(x)\right)+\epsilon_{q} \text { a.e. } x \in \Omega .
$$

Therefore, passing to the limit in this inequality, we get (3.9). 
7. Pontryagin's Principle in the Control of Variational Inequalities. In this section we will consider the following control system:

$$
\left\{\begin{array}{l}
A y+\beta(y)=f(x, y(x), u(x)) \text { in } \Omega \\
y=0 \text { on } \Gamma
\end{array}\right.
$$

where $A$ and $f$ are as in Section 2 and $\beta$ is a maximal monotone graph in $R \times R$ (see Brezis [9] and Barbu [2]) such that $\operatorname{dom}(\beta) \ni 0$. The control problem is

$$
\left(P_{\delta}\right)\left\{\begin{array}{l}
\min J(y, u)=\int_{\Omega} L(x, y(x), u(x)) d x \\
(y, u) \text { satisfies }(7.1), u(x) \in K^{\prime} \text { a.e. } x \in \Omega \text { and } g(x, y(x)) \leq \delta \forall x \in \Omega .
\end{array}\right.
$$

We keep the assumptions stated in Section 2 on the data of this problem. Then we have the following result about the state equation analogous to Theorem 2.1:

THEOREM 7.1. There exist constants $C_{5}>0$ and $\alpha \in(0,1)$ such that for every $u \in \mathcal{K}$ (7.1) has a unique solution $y_{u} \in H_{0}^{1}(\Omega) \cap C^{0, \alpha}(\bar{\Omega})$ satisfying

$$
\left\|y_{u}\right\|_{H_{0}^{1}(\Omega)}+\left\|y_{u}\right\|_{C^{0, \alpha}(\bar{\Omega})} \leq C_{5}
$$

Furthermore the mapping $u \in(\mathcal{K}, d) \longrightarrow y_{u} \in H_{0}^{1}(\Omega) \cap C^{0, \alpha}(\bar{\Omega})$ is continuous.

Proof. We may assume that $\beta(0) \ni 0$. If $\operatorname{dom}(\beta)=R$ and $\beta$ is Lipschitz and of class $C^{1}$, the result is consequence of Theorem 2.1. When $\beta$ is a general maximal monotone graph in $R \times R$ it is enough to apply the standard procedure that consists in approximating (via Yosida's approximation and convolution with a smoothing kernel: see [2]) with a Lipschitz $C^{1}$ monotone function $\beta_{c}$. In this way we obtain solutions $y_{\epsilon} \in H_{0}^{1}(\Omega) \cap C^{0 . \alpha}(\bar{\Omega})$ of

$$
\left\{\begin{array}{l}
A y+\beta_{\epsilon}(y)=f(x, y(x), u(x)) \text { in } \Omega \\
y=0 \text { on } \Gamma .
\end{array}\right.
$$

In order to pass to the limit and derive (7.2) we need a uniform estimate of $y_{\epsilon}$ in $H_{0}^{1}(\Omega) \cap C^{0, \alpha}(\bar{\Omega})$. Using the mean value theorem we can write

$$
\begin{aligned}
& \beta_{\epsilon}\left(y_{\epsilon}\right)=\beta_{\epsilon}^{\prime}\left(\hat{y}_{\epsilon}\right) y_{\epsilon} . \\
& f\left(x, y_{\epsilon}(x), u(x)\right)=f(x, 0, u(x))+\frac{\partial f}{\partial y}\left(x, \tilde{y}_{\epsilon}, u(x)\right) y_{\epsilon}
\end{aligned}
$$

with $\left|\hat{y}_{\epsilon}(x)\right| \leq\left|y_{\epsilon}(x)\right|$ and $\left|\tilde{y}_{\epsilon}(x)\right| \leq\left|y_{\epsilon}(x)\right|$ for all $x \in \Omega$. Hence

$$
\left\{\begin{array}{l}
\left.A y_{\epsilon}+\left(\beta_{\epsilon}^{\prime}\left(\hat{y}_{\epsilon}\right)(x)\right)-\frac{\partial f}{\partial y}\left(x, \tilde{y}_{\epsilon}, u(x)\right)\right) y_{\epsilon}=f(x, 0, u(x)) \text { in } \Omega \\
y=0 \text { on } \Gamma .
\end{array}\right.
$$

Now applying Lemma 2.2 to the above equation we get a uniform estimate of $y_{\epsilon}$ in $H_{0}^{1}(\Omega) \cap L^{\infty}(\Omega)$. Then the hypotheses (2.3) on $f$ imply that $\left\{f\left(x, y_{\epsilon}(x), u(x)\right)\right\}_{c>0}$ is uniform bounded in $L^{s}(\Omega)$. On the other hand, arguing as in [8, Appendix] we also 
deduce the boundedness of $\left\{\beta_{\epsilon}\left(y_{\epsilon}\right)\right\}_{\epsilon>0}$ in $L^{s}(\Omega)$. Therefore $\left\{A y_{\epsilon}\right\}_{\epsilon>0}$ is uniform bounded in $L^{s}(\Omega)$; applying Lemma 2.2 again we obtain the desired result.

The aim of this section is to prove the Pontryagin's principle for control problem $\left(P_{\delta}\right)$. For this purpose we need the following approximation scheme. First let us observe that Proposition 6.1 is still valid and consequently there exists a number $\lambda>0$ such that $\bar{u}$ is a solution of the problem

$$
\left(Q_{r}\right)\left\{\begin{array}{l}
\left.\min J_{r}(u)=\int_{\Omega} L\left(x, y_{u}(x), u(x)\right) d x+r \|\left(g\left(x, y_{u}\right)-\delta\right)^{+}\right) \|_{\infty}, \\
u \in B_{\lambda}(\bar{u}) .
\end{array}\right.
$$

Here $y_{u}$ denotes the solution of (7.1) corresponding to the control $u$.

The next step consists in approximating $\left(Q_{r}\right)$ by a new control problem with a differentiable cost functional and a state equation with a $C^{1}$ monotone term $\beta_{q}(y)$. Let us begin with the last question. Following Bonnans and Tiba [8] we will say that a maximal monotone graph in $R \times R \beta_{q}$ : with $q>1$, is an $(1 / q)$-uniform approximation to $\beta$ if $\beta_{q}$ satisfies the following two conditions:

1. $\beta(t+1 / q) \geq \beta_{q}(t) \geq \beta(t-1 / q), \forall t \in R$,

2. $\operatorname{dom}\left(\beta_{q}\right) \supset \operatorname{dom}(\beta)$.

Here we view $\beta$ and $\beta_{q}$ as multivalued operators extended on $R$ with value $-\infty$ on the left of their domains and $+\infty$ on the right of their domains, and the inequality for sets means

$$
\xi \geq \eta \geq \nu, \quad \forall \xi \in \beta(t+1 / q), \eta \in \beta_{q}(t), \nu \in \beta(t-1 / q) .
$$

A constructive procedure for $(1 / q)$-uniform approximations of class $C^{1}$ was given in [8], and the following result was proved:

Proposition 7.2. Let $u \in \mathcal{K}$. Then the problem

$$
\left\{\begin{aligned}
A y+\beta_{q}(y)=f(x, y(x), u(x)) & \text { in } \Omega \\
y=0 & \text { on } \Gamma,
\end{aligned}\right.
$$

has a unique solution $y_{q . u} \in H_{0}^{1}(\Omega) \cap C^{\alpha}(\bar{\Omega})$ and $\left\|y_{q, u}-y_{u}\right\|_{\infty} \leq 1 / q$.

Now we consider the following approximation of $\left(Q_{r}\right)$ :

$$
\left(Q_{r q}\right)\left\{\begin{array}{l}
\min J_{r, q}(u) \\
u \in B_{\lambda}(\bar{u})
\end{array}\right.
$$

where

$$
\left.J_{r . q}(u)=\int_{\Omega} L\left(x, y_{q, u}(x), u(x)\right) d x+r\left(q^{-q}+\int_{\Omega}\left[\left(g\left(x, y_{q, u}\right)-\delta\right)^{+}\right)\right]^{q} d x\right)^{1 / q} .
$$

Proposition 7.3. The following identity holds

$$
\inf \left(Q_{r}\right)=\lim _{q \rightarrow \infty} \inf \left(Q_{r, q}\right)
$$


Proof. With the aid of Proposition 7.2 we get

$$
\begin{gathered}
\left|\left\|\left(g\left(x, y_{q, u}\right)-\delta\right)^{+}\right\|_{L^{q}(\Omega)}-\left\|\left(g\left(x, y_{q, u}\right)-\delta\right)^{+}\right\|_{\infty}\right| \leq \\
\left|\left\|\left(g\left(x, y_{u}\right)-\delta\right)^{+}\right\|_{L^{q}(\Omega)}-\left\|\left(g\left(x, y_{u}\right)-\delta\right)^{+}\right\|_{\infty}\right|+ \\
\left|\left\|\left(g\left(x, y_{q, u}\right)-\delta\right)^{+}\right\|_{L^{q}(\Omega)}-\left\|\left(g\left(x, y_{u}\right)-\delta\right)^{+}\right\|_{L^{q}(\Omega)}\right|+ \\
\left|\left\|\left(g\left(x, y_{q, u}\right)-\delta\right)^{+}\right\|_{\infty}-\left\|\left(g\left(x, y_{u}\right)-\delta\right)^{+}\right\|_{\infty}\right| \leq \\
\left|\left\|\left(g\left(x, y_{u}\right)-\delta\right)^{+}\right\|_{L^{q}(\Omega)}-\left\|\left(g\left(x, y_{u}\right)-\delta\right)^{+}\right\|_{\infty}\right|+ \\
\left\|g\left(x, y_{q, u}\right)-g\left(x, y_{u}\right)\right\|_{L^{q}(\Omega)}+\left\|g\left(x, y_{q, u}\right)-g\left(x, y_{u}\right)\right\|_{\infty} \leq \\
\left|\left\|\left(g\left(x, y_{q, u}\right)-\delta\right)^{+}\right\|_{L^{q}(\Omega)}-\left\|\left(g\left(x, y_{u}\right)-\delta\right)^{+}\right\|_{\infty}\right|+\frac{m(\Omega)^{1 / q}+1}{q} \rightarrow 0 \text { as } q \rightarrow \infty
\end{gathered}
$$

Therefore we can argue as in the proof of Proposition 6.2 to deduce that

$$
\limsup _{q \rightarrow \infty} \inf \left(Q_{r, q}\right) \leq \inf \left(Q_{r}\right) \text {. }
$$

Let us prove prove the converse inequality. Let $C_{5}>0$ be the constant given in Theorem 7.1. From the properties of $g$ we obtain

$$
|g(x, t)|+\left|\frac{\partial g}{\partial y}(x, t)\right| \leq M \quad \forall(x, t) \in \bar{\Omega} \times\left[-C_{5},+C_{5}\right]
$$

for some constant $M>0$. Applying the mean value theorem and using the hypotheses (2.3), (7.4) and Proposition 7.2 we get for some constant $M^{\prime}$ and all $u \in B_{\lambda}(\bar{u})$ :

$$
\begin{gathered}
\left.\int_{\Omega} L\left(x, y_{q, u}(x), u(x)\right) d x+r\left(q^{-q}+\int_{\Omega}\left[\left(g\left(x, y_{q, u}\right)-\delta\right)^{+}\right)\right]^{q} d x\right)^{1 / q} \geq \\
\left.\int_{\Omega} L\left(x, y_{u}(x), u(x)\right) d x+r\left(q^{-q}+\int_{\Omega}\left[\left(g\left(x, y_{u}\right)-\delta\right)^{+}\right)\right]^{q} d x\right)^{1 / q}-\frac{M^{\prime}}{q} .
\end{gathered}
$$

Therefore

$$
\begin{gathered}
\liminf _{q \rightarrow \infty} \inf \left(Q_{r, q}\right) \geq \liminf _{q \rightarrow \infty} \inf \left\{\int_{\Omega} L\left(x, y_{u}(x), u(x)\right) d x+\right. \\
\left.\left.r\left(q^{-q}+\int_{\Omega}\left[\left(g\left(x, y_{u}\right)-\delta\right)^{+}\right)\right]^{q} d x\right)^{1 / q}: u \in B_{\lambda}(\bar{u})\right\} .
\end{gathered}
$$


The proof is concluded by noting that the second term of this inequality is greater than or equal to $\inf \left(Q_{r}\right)$, which is proved exactly in the same way than in Proposition 6.2. 0

Now we are ready to state the extension of Pontryagin's principle.

THEOREM 7.4 . Let $\bar{u}$ be a solution of $\left(P_{\delta}\right)$ in $(\mathcal{K}, d)$, with $\bar{y}$ its associated state. If $\left(P_{\mathcal{S}}\right)$ is strongly stable on the right, then there exist $\chi \in W^{-1, \sigma}(\Omega), \bar{p} \in W_{0}^{1, \sigma}(\Omega)$ for every $\sigma<\frac{n}{n-1}$ and $\bar{\mu} \in M(\Omega)$ such that $\chi$ is a limit point in $W^{-1, \sigma}(\Omega)$ weak of $\left\{\beta_{q}^{\prime}\left(y_{q}\right) p_{q}\right\}$ and

$$
\left\{\begin{array}{l}
A^{\star} \bar{p}+\chi=\frac{\partial f}{\partial y}(x, \bar{y}(x), \bar{u}(x))+\frac{\partial L}{\partial y}(x, \bar{y}(x), \bar{u}(x))+\frac{\partial g}{\partial y}(x, \bar{y}(x)) \bar{\mu} \text { in } \Omega \\
\bar{p}=0 \text { on } \Gamma
\end{array}\right.
$$

$$
\int_{\Omega}(\tilde{z}(x)-g(x, \bar{y}(x))) d \bar{\mu}(x) \leq 0 \quad \forall z \in C_{0}(\Omega) \text { with } z(x) \leq \delta \forall x \in \Omega,
$$

and for every $v \in K$

$$
H(x, \bar{y}(x), \bar{u}(x), \bar{p}(x)) \leq H(x, \bar{y}(x), v, \bar{p}(x)) \text { a.e. } x \in \Omega .
$$

Moreover if the conditions H1) or H2) of Theorem 3.3 hold, then

$$
H(x, \bar{y}(x), \bar{u}(x), \bar{p}(x))=\min _{v \in K} H(x, \bar{y}(x), v, \bar{p}(x)) \text { a.e. } x \in \Omega .
$$

Proof. This theorem can be proved in the same manner than Theorem 3.4: applying Proposition 7.2 we deduce that $\bar{u}$ is a solution of $\left(Q_{\tau}\right)$ and then Theorem 4.11 provides a minimum principle for an $\epsilon_{q}^{2}$-solution $u_{q}$. The adjoint state corresponding to $u_{q}$ satisfies the equation

$$
\left\{\begin{array}{l}
A^{\star} p_{q}+\beta_{q}^{\prime}\left(y_{q}\right) p_{q}=\frac{\partial f}{\partial y}\left(x, y_{q}, u_{q}\right) p_{q}+\frac{\partial L}{\partial y}\left(x, y_{q}, u_{q}\right)+\mu_{q} \frac{\partial g}{\partial y}\left(x, y_{q}\right) \text { in } \Omega \\
p_{q}=0 \text { on } \Gamma
\end{array}\right.
$$

with

$$
\mu_{q}=r\left(q^{-q}+\int_{\Omega}\left[\left(g\left(x, y_{q}(x)\right)-\delta\right)^{+}\right]^{q} d x\right)^{1 / q-1}\left[\left(g\left(x, y_{q}\right)-\delta\right)^{+}\right]^{q-1}
$$

The passage to the limit is carried out as in the proof of Theorem 3.4 with the only modification due to the term $\beta_{q}^{\prime}\left(y_{q}\right) p_{q}$. That $\left\{\mu_{q}\right\}$ is bounded in $L^{1}(\Omega)$ can be proved as in Section 7 , therefore the boundedness of $\left\{p_{q}\right\}$ in $W_{0}^{1, s}(\Omega)$ is a consequence of Lemma 2.4. Finally from the adjoint state equation it follows that $\left\{\beta_{q}^{\prime}\left(y_{q}\right) p_{q}\right\}$ is bounded in $W^{-1, \sigma}(\Omega)$, for all $\sigma<n /(n-1)$. Then there exists a subsequence, denoted in the same way, and an element $\chi \in W^{-1, \sigma}(\Omega)$ such that $\beta_{q}^{\prime}\left(y_{q}\right) p_{q} \rightarrow \chi$ weakly in $W^{-1, \sigma}(\Omega)$ when $q \rightarrow \infty$. 
REMARK 7.5. Additional information on $\lambda$ can be derincd from Theorem 7.4 for particular choices of $\beta$. For instance if $\beta$ is Lipschitz near $y_{0} \in R$ and $x_{0} \in \Omega$ is such that $y\left(x_{0}\right)=y_{0}$, then $\gamma(x) \in \partial_{c} \beta(y(x))$ with $\partial_{c} \beta$ the Clarke gradient [14] of $\beta$, for $x$ close to $x_{0}$. See e.g. [8] for other illustrations.

\section{REFERENCES}

[1] R. Addas, Sobolev Spaces, Academic Press, New York, 1975.

[2] V. Barbu. Optimal control of variational inequalities, vol. 100 of Lecture Notes in Mathematics, Pitman, London, 1984.

[3] J.F. Bosixans. El principio de Pontryagine para el control de sistemas elípticos con restricciones sobre el estado: el método de penalización, in Jornadas Hispano-Francesas sobre Control de Sistemas Distribuidos, A. Valle. ed., Málaga, 1990, 1991, pp. 13-19.

[4] - Pontryagin's principle for the optimal control of semilinear elliptic systems with state constraints, in 30th IEEE Conference on Control and Decision, Brighton, England, 1991, pp. $1976-1979$.

[5] J.F. BONNANS AND E. CASAS, Contröle de systèmes elliptiques semilinéaires comportant des contraintes sur l'état. in Nonlinear Partial Differential Equations and Their Applications. College de France Seminar. H. Brezis and J. Lions, eds., vol. 8, Longman Scientific \& Technical, New York, 1988, pp. 69-86.

[6] - Optimal control of semilinear mullistate systems with state constraints, SIAM J. on Control (t) Optim.. 27 (1989), pp. 446-455.

[†] L Lin principe de Pontryagine pour le contröle des systèmes elliptiques, J. Differential Equations. $90(1991)$, pp. $288-303$.

[8] J.F. Boxitis AND D. TIBA, Pontryagin's principle in the control of semilinear elliptic variational equations, I. Appl. Math. and Optim., 23 (1991), pp. 299-312.

[9] H. Brezis. Problèmes unilatéraux, J. Math. Pures Appl., 51 (1972), pp. 1-68.

[10] J. Burke, Calmness and exact penalization, SIAM J. on Control \& Optim., 29 (1991), pp. 493497.

[11] E. Casas. Control of an elliptic problem with pointwise state constraints, SIAM J. on Control \& Optim., 24 (1986), pp. 1309-1318.

[12] - Finte element approximations for some optimal control problems with pointwise state constraints, in IMACS '91 13th World Congress on Computation and Applied Mathematics, R. Vichnevetshy and J. Miller, eds., vol. 3, Dublin, 1991, Criterion Press, pp. 1165-1166.

[13] - Análisis de la convergencia en la aproximación numérica de problemas de control con restricciones sobre el estado, in Actas del XII C.E.D.Y.A./II Congreso de Matemática Aplicada, Oviedo, 1991, (to appear).

[14] F. Clarke, A new approach to Lagrange multipliers, Math. Op. Res., 1 (1976), pp. 165-174.

[15] I. Ekel.and, Nonconvex minimization problems, Bull. Amer. Math. Soc., 1 (1979), pp. 76-91.

[16] D. Gilbarg and N. Trudinger, Elliptic Partial Differential Equations of Second Order, Springer-Verlag, Berlin-Heidelberg-New York, 1977.

[17] Z.-X. HE, State constrained control problems governed by variational inequalities, SIAM J. Control and Optim., 25 (198T), pp. 1119-1143.

[18] J.L. Lions, Contrôle Optimal de Systèmes Gouvernés par des Equations aux Dérivées Partielles, Dunod, Paris, 1968.

[19] F. Mignot, Contrôle dans les inéquations variationelles elliptiques, Functional Analysis, 22 (1974), pp. 130-18.5. 
[20] F. Mignot and J. Puel, Optimal control in some variational inequalities, SIAM J. on Control and Optim., 22 (1984), pp. 466-476.

[21] G. Stampacchia, Le problème de Dirichlet pour les équations elliptiques du second ordre à coefficients discontinus, Ann. Inst. Fourier (Grenoble), 15 (1965), pp. 189-258. 
Imprimé en France

par

l'Institut National de Recherche en Informatique et en Automatique 
ISSN $0249-6399$ 